Keywords: $D W P F$, Glass Frit, Durability

Retention: Permanent

\title{
Frit Development for Sludge Batch 6
}

K.M. Fox

T.B. Edwards

J.R. Zamecnik

April 2010

Savannah River National Laboratory Savannah River Nuclear Solutions Aiken, SC 29808

Prepared for the U.S. Department of Energy under contract number DE-AC09-08SR22470.

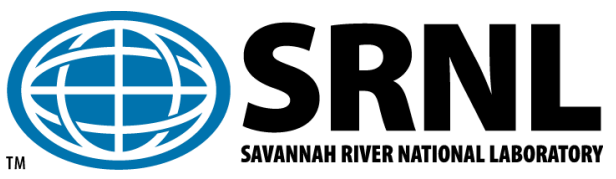


SRNL-STI-2010-00137

Revision 0

\section{DISCLAIMER}

This work was prepared under an agreement with and funded by the U.S. Government. Neither the U.S. Government or its employees, nor any of its contractors, subcontractors or their employees, makes any express or implied:

1. warranty or assumes any legal liability for the accuracy, completeness, or for the use or results of such use of any information, product, or process disclosed; or

2. representation that such use or results of such use would not infringe privately owned rights; or

3. endorsement or recommendation of any specifically identified commercial product, process, or service.

Any views and opinions of authors expressed in this work do not necessarily state or reflect those of the United States Government, or its contractors, or subcontractors.

\section{Printed in the United States of America \\ Prepared for \\ U.S. Department of Energy}




\section{REVIEWS AND APPROVALS}

AUTHORS:

K.M. Fox, Process Technology Programs

Date

T.B. Edwards, Applied Computational Engineering and Statistics

Date

J.R. Zamecnik, Engineering Process Development

Date

TECHNICAL REVIEW:

D. K. Peeler, Process Technology Programs

Date

APPROVAL:

C.C. Herman, Manager

Date

Process Technology Programs

S.L. Marra, Manager

Date

Environmental \& Chemical Process Technology Research Programs

J. E. Occhipinti, Manager

Date

Waste Solidification Engineering 
SRNL-STI-2010-00137

Revision 0

\section{EXECUTIVE SUMMARY}

The Savannah River National Laboratory (SRNL) evaluated a large number of Sludge Batch 6 (SB6) composition projections to support frit optimization for SB6 vitrification at the Defense Waste Processing Facility (DWPF). The evaluations discussed in this report occurred over a period of about 4 months, and included about 40 composition projections, developed by both Savannah River Remediation (SRR) and SRNL. Paper study assessments were used to evaluate the sludge composition projections with arrays of potential frit compositions using the predictive models in the DWPF Product Composition Control System (PCCS). Both nominal sludge compositions and sludge compositions with anticipated compositional variation were considered. The model predictions were used to identify candidate frit compositions for each SB6 projection and to provide some guidance to SRR on washing and blending strategies for SB6 preparation. This report presents a chronological review of this process and summarizes the findings at each stage.

Following initial feedback from this work, the number of washes in Tank 51 was reduced to increase the projected sodium concentration in SB6. Analyses of predicted frit performance before and after a potential decant of Tank 40 showed that the post-decant SB6 composition would be difficult to process with any frit composition and that this scenario should be avoided.

Based on the most recent SB6 projections (February 2010 SB6 composition projections developed at SRNL using the measured SB6 qualification sample composition and the revised Tank Farm washing plan), Frit 418 appears to be viable for SB6 processing at a target waste loading of $36 \%$. A Nominal Stage PCCS Measurement Acceptability Region (MAR) assessment gave projected operating windows of $25-41 \%$ waste loading, limited by predictions of nepheline crystallization. The projected operating window is reduced to $25-38 \%$ waste loading when anticipated compositional variation is considered, again limited by predictions of nepheline crystallization. Blend points between 62 and 40 inches of Sludge Batch 5 (SB5) heel in Tank 40 had no practical impact on the projected performance of Frit 418. This assessment is made from a paper study approach only and assumes that no decant of Tank 40 will occur during SB6 processing. A decant of Tank 40 would reduce the $\mathrm{Na}_{2} \mathrm{O}$ concentration in Tank 40 to a point where it would be very difficult to target a waste loading of $36 \%$ for SB6 with Frit 418.

The performance of Frit 418 with SB6 is limited by predictions of nepheline crystallization, which is a durability limiting constraint. Alternatives to Frit 418 are available that can provide equivalent projected operating windows and are limited by process related constraints (i.e., liquidus temperature predictions) rather than durability limiting constraints. A separate memorandum has recently been issued that discusses the use of Frit 418 for SB6 vitrification. ${ }^{1}$ Potential differences in melt rate among these alternative frits will be discussed in a forthcoming technical report. 


\section{TABLE OF CONTENTS}

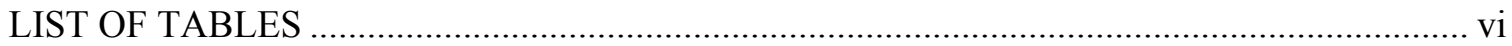

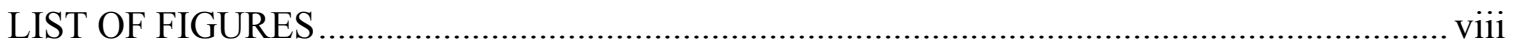

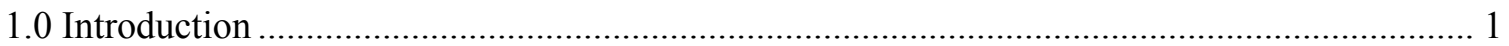

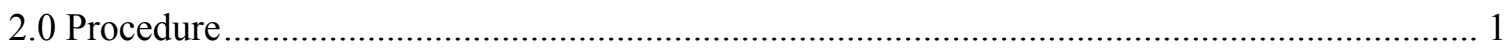

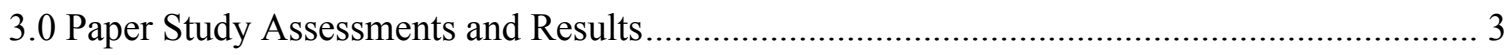

3.1 November 2009 SB6 Composition Projections ............................................................... 3

3.2 Early December 2009 SB6 Composition Projections ...................................................... 8

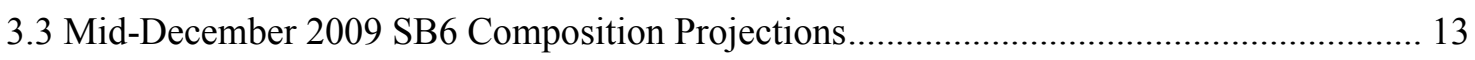

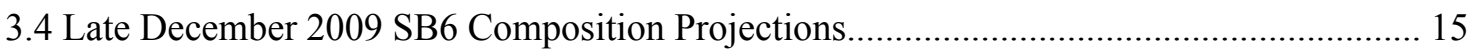

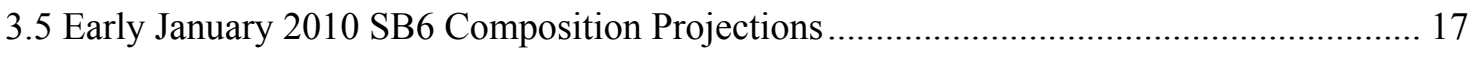

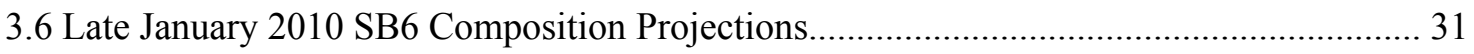

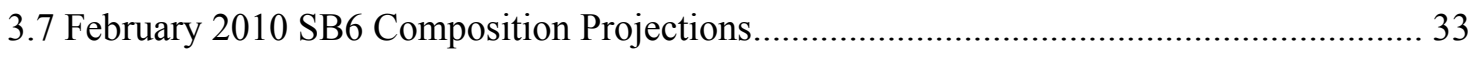

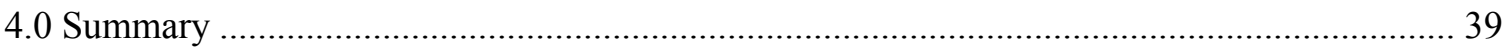

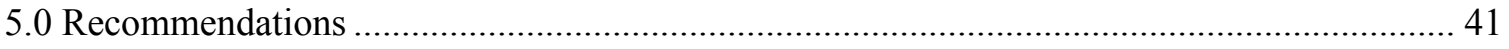

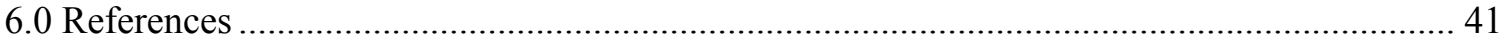




\section{LIST OF TABLES}

Table 2-1. PCCS constraints encountered during MAR assessments and their abbreviations used in this report.

Table 3-1. November 2009 SB6 Compositions from SRR. ..................................................... 3

Table 3-2. November 2009 SB6 Compositions Developed by SRNL........................................ 4

Table 3-3. Array of Frit Compositions for Nominal Stage Assessment...................................... 4

Table 3-4. Nominal Stage MAR Assessment Results for 10 Frits with the November SB6

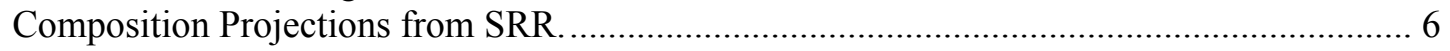

Table 3-5. Nominal Stage MAR Assessment Results for 10 Frits with the November SB6 Composition Projections from SRNL. ........................................................................ 7

Table 3-6. Sludge Composition Intervals Developed for the November SB6 Composition

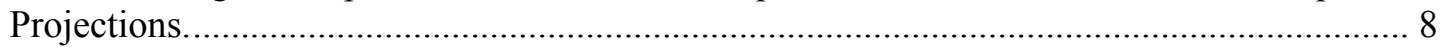

Table 3-7. Variation Stage MAR Assessment Results for the November SB6 Composition

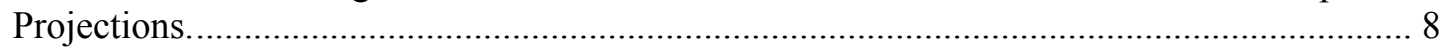

Table 3-8. Early December 2009 SRNL SB6 Composition Projections ...................................... 9

Table 3-9. Nominal Stage MAR Assessment Results for Frit 418 with the Early December 2009 SB6 Composition Projections.

Table 3-10. Nominal Stage MAR Assessment Results for Frits Down-Selected for Both the JRZ-03 and JRZ-04 Composition Projections.

Table 3-11. Nominal Stage MAR Assessment Results for Frits Down-Selected for the JRZ-04 Composition Projection.

Table 3-12. Sludge Composition Intervals Developed for the JRZ-04 Composition Projection. 12

Table 3-13. Variation Stage MAR Assessment Results for the JRZ-04 Composition Projection.12

Table 3-14. SRNL Composition Projections for Various SB6 Blend Points.

Table 3-15. Nominal Stage MAR Assessment Results for Frit 418 at Various SB6 Blend Points.

Table 3-16. SRNL SB6 Composition Projections Before and After a Decant in Tank 40.......... 16

Table 3-17. Nominal Stage MAR Assessment Results for Frit 418 with Projections JRZ-05 and JRZ-06.

Table 3-18. SB6 Composition Projections Before and After a Tank 40 Decant Using a Revised

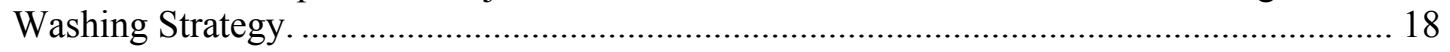

Table 3-19. Array of Frit Compositions for Nominal Stage Assessments. 18 
Table 3-20. Nominal Stage MAR Assessment Results for Frit 418 with the Early January 2010 SB6 Composition Projections.

Table 3-21. Summary of Nominal Stage Assessment Results for Individual SB6 Composition Projections. 20

Table 3-22. Frit Compositions (wt \%) for a Variation Stage Assessment with SB6 Composition Projections JRZ-07, JRZ-08 and JRZ-09.

Table 3-23. Nominal Stage MAR Assessment Results for Selected Frits Combined with Projections JRZ-07, -08 and -09, with and without ARP. 21

Table 3-24. Sludge Composition Intervals for JRZ-07, -08 and -09, with and without ARP...... 22

Table 3-25. Variation Stage Results For Selected Frits with JRZ-07, JRZ-08, and JRZ-08 and -09 Combined. 22

Table 3-26. Candidate Frit Compositions with higher $\mathrm{Na}_{2} \mathrm{O}$ Concentrations (wt \%) ................. 23

Table 3-27. Variation Stage Results for Higher $\mathrm{Na}_{2} \mathrm{O}$ Concentration Frits................................ 23

Table 3-28. Candidate Frit Compositions Similar to Frit 418 (wt \%) ........................................ 24

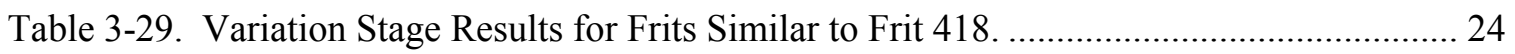

Table 3-30. Candidate Frits with Low $\mathrm{Na}_{2} \mathrm{O}$ Concentrations for JRZ-07 (wt \%)....................... 24

Table 3-31. Variation Stage Results for Low $\mathrm{Na}_{2} \mathrm{O}$ Concentration Frits with JRZ-07............... 25

Table 3-32. Higher $\mathrm{B}_{2} \mathrm{O}_{3}$ Concentration Frits for Variation Stage Assessments (wt \%)............ 25

Table 3-33. Variation Stage Assessment Results for Higher $\mathrm{B}_{2} \mathrm{O}_{3}$ Concentration Frits. ............ 26

Table 3-34. SB6 Composition Projections with Varying $\mathrm{Na}_{2} \mathrm{O}$ Concentrations.......................... 27

Table 3-35. Sludge Composition Intervals for JRZ-08b, JRZ-10 and JRZ-11........................... 28

Table 3-36. Variation Stage Assessment Results for Sludge Compositions with Varying $\mathrm{Na}_{2} \mathrm{O}$ Concentrations. 28

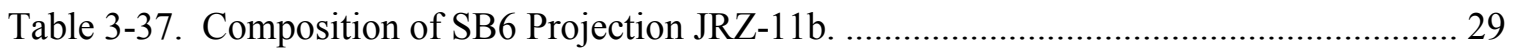

Table 3-38. Frit Compositions Selected for a Variation Stage Assessment with JRZ-11b......... 30

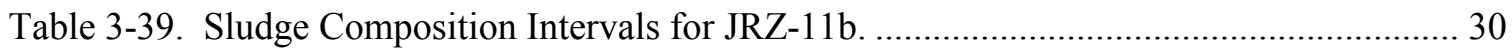

Table 3-40. Variation Stage Assessment Results for JRZ-1 1b.................................................. 31

Table 3-41. SB6 Composition Projections JanSB6-01 and JanSB6-02. ...................................... 32

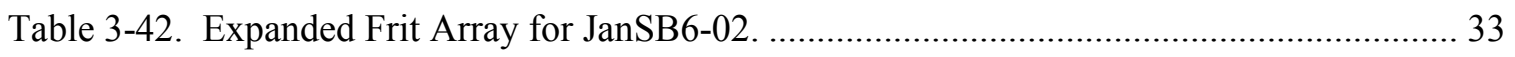

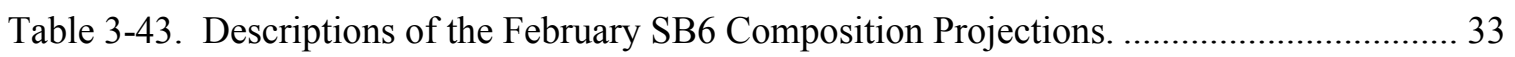

Table 3-44. Compositions of the February SB6 Projections...................................................... 34 
Table 3-45. Summary of Nominal Stage Assessment Results for Frit 418 with the FebSB6-series composition projections.

Table 3-46. Additional Frit Compositions for the FebSB6-series Projections. 35

Table 3-47. Sludge Composition Intervals for the FebSB6-series of Composition Projections. . 35

Table 3-48. Variation Stage Results for the FebSB6-series Projections with Select Frits........... 36

Table 3-49. Frits with Moderately Higher $\mathrm{Na}_{2} \mathrm{O}$ Concentrations for the FebSB6-series Projections. 36

Table 3-50. Variation Stage Results for the FebSB6-series Projections with Moderately Higher $\mathrm{Na}_{2} \mathrm{O}$ Concentration Frits. 37

Table 3-51. Frit Compositions with $\mathrm{No} \mathrm{Na}_{2} \mathrm{O}$ Selected for a Variation Stage Assessment. ........ 37

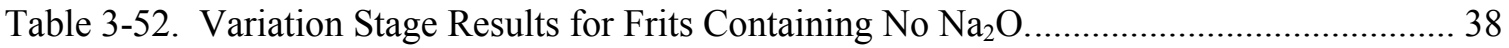

\section{LIST OF FIGURES}

Figure 2-1. Format used in this report for describing the outcome of MAR assessments (a), and a hypothetical example of MAR assessment results for a frit and sludge combination (b)....... 2 


\section{LIST OF ABBREVIATIONS}

$\begin{array}{ll}\text { ARP } & \text { Actinide Removal Process } \\ \text { DWPF } & \text { Defense Waste Processing Facility } \\ \text { EVs } & \text { Extreme Vertices } \\ \text { MAR } & \text { Measurement Acceptability Region } \\ \text { PCCS } & \text { Product Composition Control System } \\ \text { SB5 } & \text { Sludge Batch 5 } \\ \text { SB6 } & \text { Sludge Batch 6 } \\ \text { SRAT } & \text { Sludge Receipt and Adjustment Tank } \\ \text { SRNL } & \text { Savannah River National Laboratory } \\ \text { SRR } & \text { Savannah River Remediation }\end{array}$




\subsection{Introduction}

A series of composition projections for Sludge Batch 6 (SB6) was received from November 2009 through February 2010. Each of these projections was used to support paper study assessments for frit optimization, with the results being used to provide guidance on tank blending and washing plans for SB6 preparation, as well as to support the eventual recommendation of a frit composition for SB6 vitrification at the Defense Waste Processing Facility (DWPF). ${ }^{2}$ The composition projections were originally provided by Savannah River Remediation (SRR). The Savannah River National Laboratory (SRNL) developed additional composition projections to evaluate the impact of various blending and washing scenarios on frit optimization. This report presents the general procedure used for the paper study assessments, followed by a chronological discussion of the assessments of each of the SB6 composition projections and a recommended path forward.

This work was initiated under Technical Task Request HLW-DWPF-TTR-2009-0002 and Task Technical and Quality Assurance Plan SRNL-RP-2008-01342.,

\subsection{Procedure}

The approach used for the paper study evaluations was generally the same for each SB6 composition projection. First, the composition was converted to a calcined oxides basis and the concentrations of all the components were normalized to $100 \mathrm{wt} \%$. Next, the compositions were adjusted to account for additions of material from the Actinide Removal Process (ARP). ${ }^{\text {a }}$ These two steps provided a series of potential SB6 compositions for evaluation each time a new projection was received.

A Nominal Stage assessment was typically performed next, by combining each of the nominal sludge composition projections with an array of candidate frit compositions over a series of waste loadings and evaluating the resulting glass compositions against the Product Composition Control System (PCCS) Measurement Acceptability Region (MAR). The results of the Nominal Stage assessments provided projected operating windows - or waste loading intervals where the predicted properties and performance of the glass were acceptable - for each frit and sludge combination.

The format used in this report for describing the output of the paper study assessments will be as shown in Figure 2-1 (a). For each frit and sludge combination, the projected operating window will be given, along with the PCCS constraint or constraints that limit access to waste loadings outside the projected operating window. In the example shown in Figure 2-1 (b), the projected operating window is $25-42 \%$ waste loading. This means that for the example frit (Frit ABC) and sludge (Sludge XYZ) combination, the PCCS MAR assessment of the glass compositions at these waste loadings produced predicted properties that were acceptable for processing at DWPF. No constraints limited access to waste loadings down to $25 \%$, as indicated by the dash at the top of the table. The liquidus temperature constraint $\left(\mathrm{T}_{\mathrm{L}}\right)$ limited access to waste loadings of $43 \%$ and higher. A list of the limiting constraints encountered in this study and their abbreviations is given in Table 2-1. Finally, the waste loading where nepheline crystallization is predicted is given. This constraint is listed separately since nepheline crystallization can adversely impact the chemical durability of the glass waste form and must be avoided. In the example shown in Figure 2-1 (a), nepheline crystallization is predicted at waste loadings of $45 \%$ and higher.

\footnotetext{
${ }^{a}$ Compositional information for ARP additions was obtained from S.G. Subosits, "Actinide Removal Process Material Balance Calculation with Low Curie Salt Feed,” X-CLC-S-00113 Rev 0, Appendix J, September 24, 2004.
} 


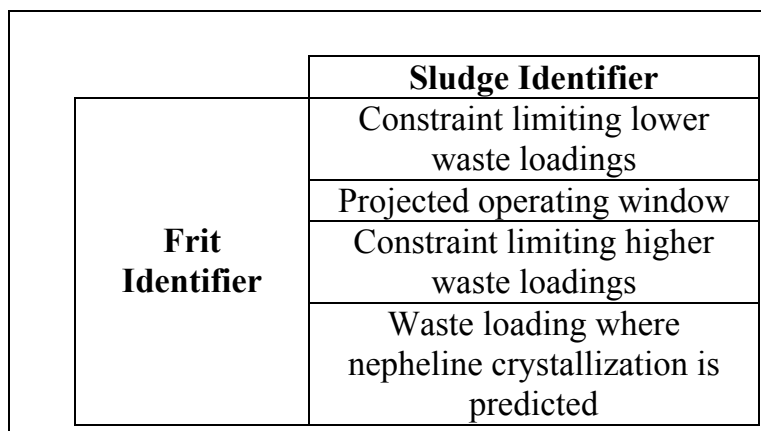

(a)

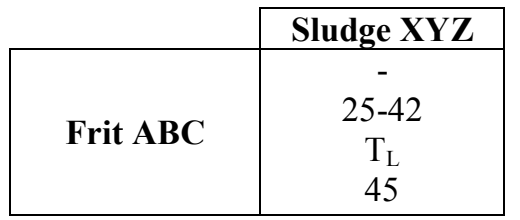

(b)

Figure 2-1. Format used in this report for describing the outcome of MAR assessments (a), and a hypothetical example of MAR assessment results for a frit and sludge combination (b).

Table 2-1. PCCS constraints encountered during MAR assessments and their abbreviations used in this report.

\begin{tabular}{||c|c||}
\hline PCCS Constraint & Abbreviation \\
\hline Poor predicted chemical durability & $\Delta \mathrm{Gp}$ \\
\hline High predicted viscosity & highv \\
\hline Low predicted viscosity & lowv \\
\hline Nepheline crystallization predicted & Neph \\
\hline High predicted liquidus temperature & $\mathrm{T}_{\mathrm{L}}$ \\
\hline
\end{tabular}

At the completion of a Nominal Stage assessment, the results were reviewed and a small number of frits that provided good projected operating windows were selected and carried forward to a Variation Stage assessment. A sludge composition region was developed for each Variation Stage assessment. This region was selected to incorporate uncertainty in the SB6 composition (usually associated with uncertainty of washing in Tank 51 and uncertainty of the Tank 40 level at the time of the SB6 blend) and anticipated compositional variation among Sludge Receipt and Adjustment Tank (SRAT) batches at DWPF. ${ }^{\mathrm{a}}$ Extreme Vertices (EVs), or corner points of this sludge region were determined using statistical analysis software. ${ }^{5}$ Each of the EVs was then combined with each of the frit compositions selected from the Nominal Stage assessment over a range of waste loadings. The resulting glass compositions were evaluated against the PCCS MAR to determine projected operating windows for each frit over which all of the EVs were predicted to be acceptable. These results are presented in the same format described in Figure 2-1.

This process was repeated for each set of SB6 compositions that was received. Any additions or modifications to this process are described along with the relevant projections in the following section.

\footnotetext{
${ }^{a}$ The variation applied was typically $7.5 \%$ for major oxide components in the sludge, and $0.5 \mathrm{wt} \%$ for minor oxide components in the sludge. The variation was increased when a sludge composition region encompassed more than a single sludge composition projection. In those cases, variation was applied to the minimum and maximum concentrations of each component among the various composition projections.
} 


\subsection{Paper Study Assessments and Results}

\subsection{November 2009 SB6 Composition Projections}

The first SB6 composition evaluated in this study was received from SRR on November 11, 2009. The composition was converted to oxides, normalized to $100 \mathrm{wt} \%$ and given the identifier NovSB6-03. During this process, it was noted that the $\mathrm{Na}_{2} \mathrm{O}$ concentration in the projection was relatively low compared with other sludge batches that have been processed at DWPF. Therefore, SRNL developed a second projection, NovSB6-04, with 2 wt \% more $\mathrm{Na}_{2} \mathrm{O}$ as an approximation of what the composition would be should SRR elect to do one less wash during preparation in Tank 51. The concentrations of the other components were normalized back to $100 \mathrm{wt} \%$. Details of these two SB6 composition projections are given in Table 3-1.

Table 3-1. November 2009 SB6 Compositions from SRR.

\begin{tabular}{|c|c|c|}
\hline Oxide (wt \%) & NovSB6-03 & NovSB6-04 \\
\hline $\mathrm{Al}_{2} \mathrm{O}_{3}$ & 25.55 & 24.87 \\
\hline $\mathrm{BaO}$ & 0.15 & 0.14 \\
\hline $\mathrm{CaO}$ & 1.55 & 1.50 \\
\hline $\mathrm{Ce}_{2} \mathrm{O}_{3}$ & 0.22 & 0.22 \\
\hline $\mathrm{Cr}_{2} \mathrm{O}_{3}$ & 0.38 & 0.37 \\
\hline $\mathrm{CuO}$ & 0.12 & 0.12 \\
\hline $\mathrm{Fe}_{2} \mathrm{O}_{3}$ & 25.86 & 25.17 \\
\hline $\mathrm{K}_{2} \mathrm{O}$ & 0.02 & 0.02 \\
\hline $\mathrm{La}_{2} \mathrm{O}_{3}$ & 0.11 & 0.11 \\
\hline $\mathrm{MgO}$ & 0.82 & 0.80 \\
\hline $\mathrm{MnO}$ & 7.48 & 7.28 \\
\hline $\mathrm{Na}_{2} \mathrm{O}$ & 25.07 & 27.07 \\
\hline $\mathrm{NiO}$ & 3.20 & 3.11 \\
\hline $\mathrm{PbO}$ & 0.03 & 0.03 \\
\hline $\mathrm{SO}_{4}{ }^{2-}$ & 1.30 & 1.27 \\
\hline $\mathrm{SiO}_{2}$ & 2.39 & 2.32 \\
\hline $\mathrm{ThO}_{2}$ & 0.02 & 0.02 \\
\hline $\mathrm{TiO}_{2}$ & 0.04 & 0.04 \\
\hline $\mathrm{U}_{3} \mathrm{O}_{8}$ & 5.23 & 5.09 \\
\hline $\mathrm{ZnO}$ & 0.10 & 0.09 \\
\hline $\mathrm{ZrO}_{2}$ & 0.37 & 0.36 \\
\hline $\begin{array}{c}\text { Supernate } \mathrm{Na}^{+} \\
(\mathrm{M})\end{array}$ & 0.796 & $\mathrm{n} / \mathrm{a}$ \\
\hline $\begin{array}{c}\text { Insoluble } \\
\text { solids (wt \%) } \\
\end{array}$ & 8.56 & $\mathrm{n} / \mathrm{a}$ \\
\hline
\end{tabular}

SRNL developed two additional SB6 composition projections using composition data from SRR and an electrolyte chemistry simulation package. ${ }^{\text {a }}$ These projections, labeled JRZ-01 and JRZ-02, represent SB6 with the baseline washing scenario and SB6 with one less wash performed, respectively. These compositions are given in Table 3-2.

\footnotetext{
${ }^{a}$ OLI Systems, Inc., Morris Plains, NJ
} 
SRNL-STI-2010-00137

Revision 0

Table 3-2. November 2009 SB6 Compositions Developed by SRNL.

\begin{tabular}{|c|c|c|}
\hline Oxide (wt \%) & JRZ-01 & JRZ-02 \\
\hline $\mathrm{Al}_{2} \mathrm{O}_{3}$ & 27.50 & 26.92 \\
\hline $\mathrm{B}_{2} \mathrm{O}_{3}$ & 0.00 & 0.00 \\
\hline $\mathrm{BaO}$ & 0.13 & 0.13 \\
\hline $\mathrm{CaO}$ & 1.89 & 1.84 \\
\hline $\mathrm{CdO}$ & 0.11 & 0.10 \\
\hline $\mathrm{Ce}_{2} \mathrm{O}_{3}$ & 0.23 & 0.23 \\
\hline $\mathrm{Cr}_{2} \mathrm{O}_{3}$ & 0.16 & 0.15 \\
\hline $\mathrm{CuO}$ & 0.11 & 0.11 \\
\hline $\mathrm{Fe}_{2} \mathrm{O}_{3}$ & 25.71 & 24.96 \\
\hline $\mathrm{Gd}_{2} \mathrm{O}_{3}$ & 0.05 & 0.05 \\
\hline $\mathrm{K}_{2} \mathrm{O}$ & 0.07 & 0.08 \\
\hline $\mathrm{La}_{2} \mathrm{O}_{3}$ & 0.11 & 0.11 \\
\hline $\mathrm{MgO}$ & 0.00 & 0.00 \\
\hline $\mathrm{MnO}$ & 7.00 & 6.79 \\
\hline $\mathrm{Na}_{2} \mathrm{O}$ & 24.33 & 26.13 \\
\hline $\mathrm{NiO}$ & 3.23 & 3.14 \\
\hline $\mathrm{PbO}$ & 0.03 & 0.03 \\
\hline $\mathrm{SO}_{4}{ }^{2-}$ & 1.38 & 1.50 \\
\hline $\mathrm{SiO}_{2}$ & 2.42 & 2.36 \\
\hline $\mathrm{SrO}$ & 0.07 & 0.07 \\
\hline $\mathrm{TiO}_{2}$ & 0.02 & 0.02 \\
\hline $\mathrm{U}_{3} \mathrm{O}_{8}$ & 5.05 & 4.90 \\
\hline $\mathrm{ZnO}$ & 0.06 & 0.06 \\
\hline $\mathrm{ZrO}_{2}$ & 0.32 & 0.31 \\
\hline $\begin{array}{l}\text { Supernate } \mathrm{Na}^{+} \\
\text {(M) }\end{array}$ & 0.765 & 0.827 \\
\hline $\begin{array}{c}\text { Insoluble } \\
\text { solids (wt \%) }\end{array}$ & 7.75 & 7.86 \\
\hline
\end{tabular}

The impact of ARP additions to each of these compositions was calculated, for a total of eight SB6 projections. The sludges were combined with an array of 6,864 potential frit compositions over waste loadings of $25-60 \%$ for a Nominal Stage MAR assessment. The minimum and maximum concentrations of each of the frit components in the array are given in Table 3-3.

Table 3-3. Array of Frit Compositions for Nominal Stage Assessment.

\begin{tabular}{|c|c|c|c|c|c||}
\hline & $\mathbf{B}_{\mathbf{2}} \mathbf{O}_{\mathbf{3}}$ & $\mathbf{C a O}$ & $\mathbf{L i}_{\mathbf{2}} \mathbf{O}$ & $\mathbf{N a}_{2} \mathbf{O}$ & $\mathbf{S i O}_{\mathbf{2}}$ \\
\hline $\begin{array}{c}\text { Minimum } \\
(\text { wt \%) }\end{array}$ & 8.0 & 0.0 & 5.0 & 2.0 & 51.0 \\
\hline $\begin{array}{c}\text { Maximum } \\
(\text { wt \%) }\end{array}$ & 20.0 & 5.0 & 12.0 & 12.0 & 85.0 \\
\hline
\end{tabular}

The Nominal Stage assessment identified 407 frit compositions that gave projected operating windows of at least $28-40 \%$ for all eight of the sludge compositions. Note that the sulfate concentration constraint was ignored throughout this study due to uncertainty in the final SB6 composition. The data were reviewed and 10 frits of interest (including Frit $418^{\mathrm{a}}$ ) were down-

${ }^{a}$ Frit 418 is included throughout this study since it is currently used for Sludge Batch 5 (SB5) vitrification and it may be advantageous to continue into SB6 processing without changing frit compositions. 
selected for further study. The Nominal Stage results for these 10 frits with the SRR composition projections are given in Table 3-4, and the results with the SRNL composition projections are given in Table 3-5. The projected operating windows are limited to maximum waste loadings of $40-44 \%$ for the various frits. The limiting constraints are dependent on the frit composition. In general, frits with low $\mathrm{Na}_{2} \mathrm{O}$ concentrations result in projected operating windows that are $\mathrm{T}_{\mathrm{L}}$ or low viscosity limited, while frits with higher $\mathrm{Na}_{2} \mathrm{O}$ concentrations resulted in nepheline limited systems. 
Table 3-4. Nominal Stage MAR Assessment Results for 10 Frits with the November SB6 Composition Projections from SRR.

\begin{tabular}{|c|c|c|c|c|}
\hline $\begin{array}{c}\text { Frit Oxides } \\
(w t \%)\end{array}$ & NovSB6-03 & NovSB6-03ARP & NovSB6-04 & NovSB6-04ARP \\
\hline $\begin{array}{l}\text { B-10;Ca-3;Li-11; } \\
\text { Na-2;Si-74 }\end{array}$ & $\begin{array}{c}- \\
25-40 \\
\mathrm{~T}_{\mathrm{L}} \\
46 \\
\end{array}$ & $\begin{array}{c}- \\
25-42 \\
\mathrm{~T}_{\mathrm{L}} \\
46 \\
\end{array}$ & $\begin{array}{c}- \\
25-42 \\
\text { lowv } \\
46\end{array}$ & $\begin{array}{c}- \\
25-41 \\
\text { lowv } \\
46\end{array}$ \\
\hline $\begin{array}{l}\text { B-12;Ca-3;Li-9; } \\
\text { Na-4;Si-72 }\end{array}$ & $\begin{array}{c}- \\
25-40 \\
\mathrm{~T}_{\mathrm{L}} \\
44\end{array}$ & $\begin{array}{c}- \\
25-42 \\
\mathrm{~T}_{\mathrm{L}} \\
44\end{array}$ & $\begin{array}{c}- \\
25-42 \\
\text { lowv } \\
44\end{array}$ & $\begin{array}{c}- \\
25-41 \\
\text { lowv } \\
44\end{array}$ \\
\hline $\begin{array}{l}\text { B-12;Li-11; } \\
\text { Na-2;Si-75 }\end{array}$ & $\begin{array}{c}- \\
25-40 \\
\mathrm{~T}_{\mathrm{L}} \\
47\end{array}$ & $\begin{array}{c}- \\
25-42 \\
\mathrm{~T}_{\mathrm{L}} \\
47\end{array}$ & $\begin{array}{c}- \\
25-41 \\
\text { lowv } \\
46\end{array}$ & $\begin{array}{c}- \\
25-40 \\
\text { lowv } \\
46\end{array}$ \\
\hline $\begin{array}{l}\text { B-14;Li-9; } \\
\text { Na-4;Si-73 }\end{array}$ & $\begin{array}{c}- \\
25-40 \\
\mathrm{~T}_{\mathrm{L}} \\
45 \\
\end{array}$ & $\begin{array}{c}- \\
25-42 \\
\mathrm{~T}_{\mathrm{L}} \\
45 \\
\end{array}$ & $\begin{array}{c}- \\
25-41 \\
\text { lowv } \\
44 \\
\end{array}$ & $\begin{array}{c}- \\
25-40 \\
\text { lowv } \\
44\end{array}$ \\
\hline $\begin{array}{l}\text { B-8;Ca-3;Li-12; } \\
\text { Na-2;Si-75 }\end{array}$ & $\begin{array}{c}- \\
25-41 \\
\mathrm{~T}_{\mathrm{L}} \\
47 \\
\end{array}$ & $\begin{array}{c}- \\
25-42 \\
\text { lowv } \\
47 \\
\end{array}$ & $\begin{array}{c}- \\
25-41 \\
\text { lowv } \\
46 \\
\end{array}$ & $\begin{array}{c}- \\
25-40 \\
\text { lowv } \\
46 \\
\end{array}$ \\
\hline $\begin{array}{l}\text { B-8;Ca-3;Li-8; } \\
\text { Na-8;Si-73 }\end{array}$ & $\begin{array}{c}- \\
25-41 \\
\text { Neph } \\
42\end{array}$ & $\begin{array}{c}- \\
25-41 \\
\text { Neph } \\
42\end{array}$ & $\begin{array}{c}- \\
25-40 \\
\text { Neph } \\
41\end{array}$ & $\begin{array}{c}- \\
25-40 \\
\text { Neph } \\
41\end{array}$ \\
\hline $\begin{array}{l}\text { B-8;Li-12; } \\
\text { Na-2;Si-78 }\end{array}$ & $\begin{array}{c}- \\
25-42 \\
\mathrm{~T}_{\mathrm{L}} \\
48\end{array}$ & $\begin{array}{c}- \\
25-44 \\
\mathrm{~T}_{\mathrm{L}} \\
48\end{array}$ & $\begin{array}{c}- \\
25-44 \\
\text { lowv } \\
47\end{array}$ & $\begin{array}{c}- \\
25-43 \\
\text { lowv } \\
47\end{array}$ \\
\hline $\begin{array}{c}\text { B-8;Li-8; } \\
\text { Na-8;Si-76 } \\
\text { (Frit 418) }\end{array}$ & $\begin{array}{c}- \\
25-42 \\
\text { Neph } \\
43 \\
\end{array}$ & $\begin{array}{c}- \\
25-42 \\
\text { Neph } \\
43 \\
\end{array}$ & $\begin{array}{c}- \\
25-42 \\
\text { Neph } \\
43 \\
\end{array}$ & $\begin{array}{c}- \\
25-42 \\
\text { Neph } \\
43 \\
\end{array}$ \\
\hline $\begin{array}{l}\text { B-9;Ca-3;Li-10; } \\
\text { Na-4;Si-74 }\end{array}$ & $\begin{array}{c}- \\
25-41 \\
\mathrm{~T}_{\mathrm{L}} \\
45\end{array}$ & $\begin{array}{c}- \\
25-43 \\
\mathrm{~T}_{\mathrm{L}} \\
45\end{array}$ & $\begin{array}{c}- \\
25-43 \\
\text { lowv, Neph } \\
44\end{array}$ & $\begin{array}{c}- \\
25-42 \\
\text { lowv } \\
44\end{array}$ \\
\hline $\begin{array}{l}\text { B-9;Li-10; } \\
\text { Na-4;Si-77 }\end{array}$ & $\begin{array}{c}- \\
25-42 \\
\mathrm{~T}_{\mathrm{L}} \\
46\end{array}$ & $\begin{array}{c}- \\
25-44 \\
\mathrm{~T}_{\mathrm{L}} \\
46\end{array}$ & $\begin{array}{c}- \\
25-44 \\
\text { Neph } \\
45\end{array}$ & $\begin{array}{c}- \\
25-44 \\
\text { lowv } \\
46\end{array}$ \\
\hline
\end{tabular}


Table 3-5. Nominal Stage MAR Assessment Results for 10 Frits with the November SB6 Composition Projections from SRNL.

\begin{tabular}{|c|c|c|c|c|}
\hline $\begin{array}{c}\text { Frit Oxides } \\
(\text { wt \%) }\end{array}$ & JRZ-01 & JRZ-01ARP & JRZ-02 & JRZ-02ARP \\
\hline $\begin{array}{l}\text { B-10;Ca-3;Li-11; } \\
\text { Na-2;Si-74 }\end{array}$ & $\begin{array}{c}- \\
25-44 \\
\mathrm{~T}_{\mathrm{L}} \\
46\end{array}$ & $\begin{array}{c}- \\
25-45 \\
\text { Neph } \\
46\end{array}$ & $\begin{array}{c}- \\
25-44 \\
\text { Neph } \\
45\end{array}$ & $\begin{array}{c}25-43 \\
\text { lowv } \\
45\end{array}$ \\
\hline $\begin{array}{l}\text { B-12;Ca-3;Li-9; } \\
\text { Na-4;Si-72 }\end{array}$ & $\begin{array}{c}- \\
25-43 \\
\mathrm{~T}_{\mathrm{L}}, \text { Neph } \\
44\end{array}$ & $\begin{array}{c}- \\
25-43 \\
\text { Neph } \\
44 \\
\end{array}$ & $\begin{array}{c}- \\
25-42 \\
\text { Neph } \\
43 \\
\end{array}$ & $\begin{array}{c}- \\
25-42 \\
\text { Neph } \\
43 \\
\end{array}$ \\
\hline $\begin{array}{l}\text { B-12;Li-11; } \\
\text { Na-2;Si-75 }\end{array}$ & $\begin{array}{c}- \\
25-44 \\
\mathrm{~T}_{\mathrm{L}} \\
46 \\
\end{array}$ & $\begin{array}{c}- \\
25-45 \\
\text { lowv, Neph } \\
46 \\
\end{array}$ & $\begin{array}{c}- \\
25-44 \\
\text { lowv, Neph } \\
45 \\
\end{array}$ & $\begin{array}{c}25-43 \\
\text { lowv } \\
45\end{array}$ \\
\hline $\begin{array}{l}\text { B-14;Li-9; } \\
\text { Na-4;Si-73 }\end{array}$ & $\begin{array}{c}- \\
25-43 \\
\mathrm{~T}_{\mathrm{L}}, \mathrm{Neph} \\
44\end{array}$ & $\begin{array}{c}- \\
25-43 \\
\text { Neph } \\
44 \\
\end{array}$ & $\begin{array}{c}- \\
25-42 \\
\text { Neph } \\
43 \\
\end{array}$ & $\begin{array}{c}- \\
25-43 \\
\text { lowv, Neph } \\
44 \\
\end{array}$ \\
\hline $\begin{array}{l}\text { B-8;Ca-3;Li-12; } \\
\text { Na-2;Si-75 }\end{array}$ & $\begin{array}{c}- \\
25-45 \\
\mathrm{~T}_{\mathrm{L}}, \text { Neph } \\
46\end{array}$ & $\begin{array}{c}- \\
25-45 \\
\text { lowv, Neph } \\
46 \\
\end{array}$ & $\begin{array}{c}- \\
25-44 \\
\text { lowv, Neph } \\
45 \\
\end{array}$ & $\begin{array}{c}- \\
25-43 \\
\text { lowv } \\
45 \\
\end{array}$ \\
\hline $\begin{array}{l}\text { B-8;Ca-3;Li-8; } \\
\text { Na-8;Si-73 }\end{array}$ & $\begin{array}{c}- \\
25-40 \\
\text { Neph } \\
41 \\
\end{array}$ & $\begin{array}{c}- \\
25-41 \\
\text { Neph } \\
42 \\
\end{array}$ & $\begin{array}{c}- \\
25-40 \\
\text { Neph } \\
41 \\
\end{array}$ & $\begin{array}{c}- \\
25-40 \\
\text { Neph } \\
41 \\
\end{array}$ \\
\hline $\begin{array}{l}\text { B-8;Li-12; } \\
\text { Na-2;Si-78 }\end{array}$ & $\begin{array}{c}- \\
25-45 \\
\mathrm{~T}_{\mathrm{L}} \\
47 \\
\end{array}$ & $\begin{array}{c}- \\
25-46 \\
\text { Neph } \\
47 \\
\end{array}$ & $\begin{array}{c}- \\
25-45 \\
\text { Neph } \\
46 \\
\end{array}$ & $\begin{array}{c}- \\
25-45 \\
\text { lowv } \\
47 \\
\end{array}$ \\
\hline $\begin{array}{c}\text { B-8;Li-8; } \\
\text { Na-8;Si-76 } \\
\text { (Frit 418) }\end{array}$ & $\begin{array}{c}- \\
25-42 \\
\text { Neph } \\
43 \\
\end{array}$ & $\begin{array}{c}- \\
25-42 \\
\text { Neph } \\
43\end{array}$ & $\begin{array}{c}- \\
25-41 \\
\text { Neph } \\
42 \\
\end{array}$ & $\begin{array}{c}- \\
25-41 \\
\text { Neph } \\
42 \\
\end{array}$ \\
\hline $\begin{array}{l}\text { B-9;Ca-3;Li-10; } \\
\text { Na-4;Si-74 }\end{array}$ & $\begin{array}{c}- \\
25-43 \\
\text { Neph } \\
44 \\
\end{array}$ & $\begin{array}{c}- \\
25-44 \\
\text { Neph } \\
45 \\
\end{array}$ & $\begin{array}{c}- \\
25-43 \\
\text { Neph } \\
44 \\
\end{array}$ & $\begin{array}{c}25-43 \\
\text { Neph } \\
44\end{array}$ \\
\hline $\begin{array}{l}\text { B-9;Li-10; } \\
\text { Na-4;Si-77 }\end{array}$ & $\begin{array}{c}- \\
25-45 \\
\mathrm{~T}_{\mathrm{L}}, \mathrm{Neph} \\
46\end{array}$ & $\begin{array}{c}- \\
25-45 \\
\text { Neph } \\
46 \\
\end{array}$ & $\begin{array}{c}- \\
25-44 \\
\text { Neph } \\
45 \\
\end{array}$ & $\begin{array}{c}- \\
25-44 \\
\text { Neph } \\
45 \\
\end{array}$ \\
\hline
\end{tabular}

Sludge composition intervals were developed for a Variation Stage assessment encompassing the eight November sludge composition projections, as shown in Table 3-6. The EVs of this region were then identified and combined with the 10 frit compositions over waste loadings of $25-50 \%$. Each glass composition was then evaluated against the PCCS MAR, and the results are given in Table 3-7. 
Table 3-6. Sludge Composition Intervals Developed for the November SB6 Composition Projections.

\begin{tabular}{|c|c|c|c|c|c|c||}
\hline Oxide & $\mathbf{A l}_{2} \mathbf{O}_{\mathbf{3}}$ & $\mathbf{C a O}$ & $\mathbf{F e}_{2} \mathbf{O}_{\mathbf{3}}$ & $\mathbf{M g O}$ & $\mathbf{M n O}$ & $\mathbf{N a}_{\mathbf{2}} \mathbf{O}$ \\
\hline Minimum (wt \%) & 22.15 & 0.98 & 22.5 & 0.00 & 6.18 & 22.51 \\
\hline Maximum (wt \%) & 29.56 & 2.39 & 27.80 & 1.32 & 8.04 & 29.79 \\
\hline \multicolumn{7}{|l|}{} \\
\hline Oxide & $\mathbf{N i O}$ & $\mathbf{S O}_{\mathbf{4}}{ }^{2-}$ & $\mathbf{S i O}_{\mathbf{2}}$ & $\mathbf{T i O}_{\mathbf{2}}$ & $\mathbf{U}_{\mathbf{3}} \mathbf{O}_{\mathbf{8}}$ & Others $^{\mathrm{a}}$ \\
\hline Minimum (wt \%) & 2.53 & 1.02 & 1.74 & 0.00 & 4.32 & 0.62 \\
\hline Maximum (wt \%) & 3.73 & 2.00 & 2.92 & 1.84 & 5.73 & 2.11 \\
\hline
\end{tabular}

Table 3-7. Variation Stage MAR Assessment Results for the November SB6 Composition Projections.

\begin{tabular}{|c|c|c|c|c|c|}
\hline $\begin{array}{l}\text { Frit Oxides } \\
\text { (wt \%) }\end{array}$ & $\begin{array}{c}\text { B-10;Ca-3; } \\
\text { Li-11;Na-2; } \\
\text { Si-74 }\end{array}$ & $\begin{array}{l}\text { B-12;Ca-3; } \\
\text { Li-9;Na-4; } \\
\text { Si-72 }\end{array}$ & $\begin{array}{l}\text { B-12;Li-11; } \\
\text { Na-2;Si-75 }\end{array}$ & $\begin{array}{l}\text { B-14;Li-9; } \\
\text { Na-4;Si-73 }\end{array}$ & $\begin{array}{c}\text { B-8;Ca-3; } \\
\text { Li-12;Na-2; } \\
\text { Si-75 }\end{array}$ \\
\hline $\begin{array}{c}\text { Variation } \\
\text { Stage MAR } \\
\text { Outcome }\end{array}$ & $\begin{array}{c}25-34 \\
\mathrm{~T}_{\mathrm{L}} \\
42 \\
\end{array}$ & $\begin{array}{c}- \\
25-33 \\
\mathrm{~T}_{\mathrm{L}} \\
40 \\
\end{array}$ & $\begin{array}{c}25-33 \\
\mathrm{~T}_{\mathrm{L}} \\
41 \\
\end{array}$ & $\begin{array}{c}25-33 \\
\mathrm{~T}_{\mathrm{L}} \\
41 \\
\end{array}$ & $\begin{array}{c}25-35 \\
\mathrm{~T}_{\mathrm{L}} \\
42 \\
\end{array}$ \\
\hline $\begin{array}{l}\text { Frit Oxides } \\
\text { (wt \%) }\end{array}$ & $\begin{array}{l}\text { B-8;Ca-3;Li-8; } \\
\text { Na-8;Si-73 }\end{array}$ & $\begin{array}{l}\text { B-8;Li-12; } \\
\text { Na-2;Si-78 }\end{array}$ & $\begin{array}{c}\text { B-8;Li-8; } \\
\text { Na-8;Si-76 } \\
\text { (Frit 418) }\end{array}$ & $\begin{array}{c}\text { B-9;Ca-3; } \\
\text { Li-10;Na-4; } \\
\text { Si-74 }\end{array}$ & $\begin{array}{l}\text { B-9;Li-10; } \\
\text { Na-4;Si-77 }\end{array}$ \\
\hline $\begin{array}{c}\text { Variation } \\
\text { Stage MAR } \\
\text { Outcome }\end{array}$ & $\begin{array}{c}- \\
25-35 \\
\Delta \mathrm{Gp} \\
38\end{array}$ & $\begin{array}{c}- \\
25-35 \\
\mathrm{~T}_{\mathrm{L}} \\
43\end{array}$ & $\begin{array}{c}- \\
25-37 \\
\mathrm{~T}_{\mathrm{L}} \\
39\end{array}$ & $\begin{array}{c}- \\
25-35 \\
\mathrm{~T}_{\mathrm{L}} \\
41\end{array}$ & $\begin{array}{c}- \\
25-35 \\
\mathrm{~T}_{\mathrm{L}} \\
42\end{array}$ \\
\hline
\end{tabular}

The results in Table 3-7 show that Frit 418 provides the largest projected operating window over which all of the EVs produce acceptable glasses for this sludge region. However, the projected operating window is limited to a relatively low $37 \%$ waste loading before some of the EV combinations begin to fail the $\mathrm{T}_{\mathrm{L}}$ constraint. Nepheline is predicted for some of the EVs at $39 \%$ waste loading. While one frit is limited by predictions of poor durability $(\Delta \mathrm{Gp})$, all of the other frits are $T_{L}$ limited at the Variation Stage for this sludge region. Note that the projected operating windows for these frits are significantly reduced when variation is considered as compared with the Nominal Stage assessment results (Table 3-4 and Table 3-5).

\subsection{Early December 2009 SB6 Composition Projections}

SRNL completed two SB6 composition projections on December 7, 2009, which better reflected the planned washing and decanting strategy in Tank 51. Specifically, the projections included a larger volume Decant $\mathrm{L}$ at the end of the washing process to increase the solids loading of the SB6 batch. The first composition projection, JRZ-03, represents the baseline washing strategy, while JRZ-04 represents a strategy with one less wash in Tank 51. Both projections have lower $\mathrm{Na}_{2} \mathrm{O}$ concentrations as compared to JRZ-01 and JRZ-02. The projections are detailed in Table 3-8.

\footnotetext{
${ }^{a}$ The Others group consists of oxides present at concentrations that are below reportable limits for DWPF (e.g., BaO, $\mathrm{CdO}, \mathrm{Ce}_{2} \mathrm{O}_{3}, \mathrm{Cr}_{2} \mathrm{O}_{3}, \mathrm{CuO}, \mathrm{Gd}_{2} \mathrm{O}_{3}, \mathrm{~K}_{2} \mathrm{O}, \mathrm{La}_{2} \mathrm{O}_{3}, \mathrm{MoO}_{3}, \mathrm{P}_{2} \mathrm{O}_{5}, \mathrm{PbO}, \mathrm{SrO}, \mathrm{ZnO}$ and $\mathrm{ZrO}_{2}$ ).
} 
SRNL-STI-2010-00137

Revision 0

Table 3-8. Early December 2009 SRNL SB6 Composition Projections

\begin{tabular}{|c|c|c|}
\hline Oxide (wt \%) & JRZ-03 & JRZ-04 \\
\hline $\mathrm{Al}_{2} \mathrm{O}_{3}$ & 28.96 & 28.42 \\
\hline $\mathrm{B}_{2} \mathrm{O}_{3}$ & 0.00 & 0.00 \\
\hline $\mathrm{BaO}$ & 0.14 & 0.14 \\
\hline $\mathrm{CaO}$ & 2.03 & 1.99 \\
\hline $\mathrm{CdO}$ & 0.11 & 0.11 \\
\hline $\mathrm{Ce}_{2} \mathrm{O}_{3}$ & 0.26 & 0.26 \\
\hline $\mathrm{Cr}_{2} \mathrm{O}_{3}$ & 0.16 & 0.16 \\
\hline $\mathrm{CuO}$ & 0.12 & 0.12 \\
\hline $\mathrm{Fe}_{2} \mathrm{O}_{3}$ & 27.66 & 26.98 \\
\hline $\mathrm{Gd}_{2} \mathrm{O}_{3}$ & 0.06 & 0.06 \\
\hline $\mathrm{K}_{2} \mathrm{O}$ & 0.06 & 0.06 \\
\hline $\mathrm{La}_{2} \mathrm{O}_{3}$ & 0.12 & 0.10 \\
\hline $\mathrm{MgO}$ & 0.76 & 0.74 \\
\hline $\mathrm{MnO}$ & 7.53 & 7.33 \\
\hline $\mathrm{Na}_{2} \mathrm{O}$ & 18.90 & 20.58 \\
\hline $\mathrm{NiO}$ & 3.47 & 3.38 \\
\hline $\mathrm{PbO}$ & 0.03 & 0.03 \\
\hline $\mathrm{SO}_{4}{ }^{2-}$ & 1.07 & 1.16 \\
\hline $\mathrm{SiO}_{2}$ & 2.62 & 2.55 \\
\hline $\mathrm{SrO}$ & 0.08 & 0.07 \\
\hline $\mathrm{TiO}_{2}$ & 0.02 & 0.02 \\
\hline $\mathrm{U}_{3} \mathrm{O}_{8}$ & 5.43 & 5.30 \\
\hline $\mathrm{ZnO}$ & 0.06 & 0.06 \\
\hline $\mathrm{ZrO}_{2}$ & 0.35 & 0.35 \\
\hline $\begin{array}{c}\text { Supernate } \mathrm{Na}^{+} \\
(\mathrm{M})\end{array}$ & 0.751 & 0.800 \\
\hline $\begin{array}{c}\text { Insoluble solids } \\
\text { (wt \%) }\end{array}$ & 10.52 & 10.44 \\
\hline
\end{tabular}

The impact of ARP additions was calculated, resulting in a total of four SB6 projections. Each of these sludge compositions was combined with the array of 6,864 potential frit compositions given earlier in Table 3-3 over waste loadings of 25-60\% for a Nominal Stage MAR assessment. The results were screened to identify frits that provided projected operating windows of at least 28$40 \% \mathrm{WL}$ for each of the projections, with 317 frits meeting this criterion for JRZ-03, 665 frits for JRZ-03 with ARP, 658 frits for JRZ-04 and 1040 frits for JRZ-04 with ARP.

The Nominal Stage results for Frit 418 with the four early December SB6 composition projections are given in Table 3-9. The systems are generally limited by liquidus temperature predictions at about $40 \%$ WL. Frit 418 with JRZ-04ARP has a slightly larger projected operating window that is limited by both liquidus temperature and nepheline crystallization predictions. 
Table 3-9. Nominal Stage MAR Assessment Results for Frit 418 with the Early December 2009 SB6 Composition Projections.

\begin{tabular}{|c|c|c|c|c||}
\hline $\begin{array}{c}\text { Frit Oxides } \\
\text { (wt \%) }\end{array}$ & JRZ-03 & JRZ-03ARP & JRZ-04 & JRZ-04ARP \\
\hline B-8;Li-8; & - & - & - & - \\
Na-8;Si-76 & $25-39$ & $25-41$ & $25-41$ & $25-43$ \\
(Frit 418) & $\mathrm{T}_{\mathrm{L}}$ & $\mathrm{T}_{\mathrm{L}}$ & $\mathrm{T}_{\mathrm{L}}$ & $\mathrm{T}_{\mathrm{L}}, \mathrm{Neph}$ \\
& 45 & 45 & 44 & 44 \\
\hline
\end{tabular}

Two groups of frits were down-selected from the Nominal Stage results for further study. The first group of frits provides reasonable projected operating windows for both JRZ-03 and JRZ-04, with and without ARP. The compositions and results of the Nominal Stage MAR assessment for these frits are given in Table 3-10.

Table 3-10. Nominal Stage MAR Assessment Results for Frits Down-Selected for Both the JRZ-03 and JRZ-04 Composition Projections.

\begin{tabular}{|c|c|c|c|c||}
\hline $\begin{array}{c}\text { Frit Oxides } \\
\text { (wt \%) }\end{array}$ & JRZ-03 & JRZ-03ARP & JRZ-04 & JRZ-04ARP \\
\hline & - & - & - & - \\
B-8;Li-11; & $25-40$ & $25-42$ & $25-42$ & $25-44$ \\
Na-6;Si-75 & $\mathrm{T}_{\mathrm{L}}$ & $\mathrm{T}_{\mathrm{L}}$ & $\mathrm{T}_{\mathrm{L}}$ & $\mathrm{T}_{\mathrm{L}}, \mathrm{Neph}$ \\
& 46 & 46 & 45 & 45 \\
\hline & - & - & - & - \\
B-10;Li-11; & $25-40$ & $25-42$ & $25-42$ & $25-42$ \\
Na-6;Si-73 & $\mathrm{T}_{\mathrm{L}}$ & $\mathrm{T}_{\mathrm{L}}$ & $\mathrm{T}_{\mathrm{L}}$ & lowv \\
& 45 & 45 & 44 & 44 \\
\hline & - & - & - & - \\
B-8;Ca-3;Li-11; & $25-40$ & $25-42$ & $25-42$ & $25-43$ \\
Na-6;Si-72 & $\mathrm{T}_{\mathrm{L}}$ & $\mathrm{T}_{\mathrm{L}}$ & $\mathrm{T}_{\mathrm{L}}$ & $10 w \mathrm{v}, \mathrm{Neph}$ \\
& 44 & 44 & 44 & 44 \\
\hline & - & - & - & - \\
B-8;Li-10; & $25-41$ & $25-43$ & $25-42$ & $25-42$ \\
Na-8;Si-74 & $\mathrm{T}_{\mathrm{L}}$ & $\mathrm{T}_{\mathrm{L}}, \mathrm{Neph}$ & Neph & Neph \\
& 44 & 44 & 43 & 43 \\
\hline & - & - & - & - \\
B-12;Li-9; & $25-40$ & $25-42$ & $25-41$ & $25-41$ \\
Na-8;Si-71 & $\mathrm{T}_{\mathrm{L}}$ & $\mathrm{T}_{\mathrm{L}}, \mathrm{Neph}$ & Neph & Neph \\
& 43 & 43 & 42 & 42 \\
\hline \multirow{2}{*}{} & - & - & - & - \\
B-8;Ca-3;Li-10; & $25-41$ & $25-42$ & $25-41$ & $25-41$ \\
Na-8;Si-71 & $\mathrm{T}_{\mathrm{L}}$ & Neph & Neph & Neph \\
& 43 & 43 & 42 & 42 \\
\hline
\end{tabular}

The second group of frits provides reasonable projected operating windows for JRZ-04 with and without ARP only. The compositions and results of the Nominal Stage MAR assessment for these frits are given in Table 3-11. 
Table 3-11. Nominal Stage MAR Assessment Results for Frits Down-Selected for the JRZ-04 Composition Projection.

\begin{tabular}{|c|c|c|}
\hline $\begin{array}{c}\text { Frit Oxides } \\
(w t \%)\end{array}$ & JRZ-04 & JRZ-04ARP \\
\hline $\begin{array}{l}\text { B-8;Li-11; } \\
\text { Na-6;Si-75 }\end{array}$ & $\begin{array}{c}- \\
25-42 \\
\mathrm{~T}_{\mathrm{L}} \\
45\end{array}$ & $\begin{array}{c}- \\
25-44 \\
\mathrm{~T}_{\mathrm{L}}, \mathrm{Neph} \\
45\end{array}$ \\
\hline $\begin{array}{l}\text { B-14;Li-9; } \\
\text { Na-6;Si-71 }\end{array}$ & $\begin{array}{c}- \\
25-40 \\
\mathrm{~T}_{\mathrm{L}} \\
43\end{array}$ & $\begin{array}{c}- \\
25-42 \\
\mathrm{~T}_{\mathrm{L}}, \mathrm{Neph} \\
43\end{array}$ \\
\hline $\begin{array}{l}\text { B-8;Ca-3;Li-11; } \\
\text { Na-6;Si-72 }{ }^{\dagger}\end{array}$ & $\begin{array}{c}- \\
25-42 \\
\mathrm{~T}_{\mathrm{L}} \\
44 \\
\end{array}$ & $\begin{array}{c}- \\
25-43 \\
\text { lowv, Neph } \\
44\end{array}$ \\
\hline $\begin{array}{c}\text { B-12;Ca-3;Li-9; } \\
\text { Na-6;Si-70 }\end{array}$ & $\begin{array}{c}- \\
25-40 \\
\mathrm{~T}_{\mathrm{L}} \\
43 \\
\end{array}$ & $\begin{array}{c}- \\
25-42 \\
\mathrm{~T}_{\mathrm{L}}, \mathrm{Neph} \\
43 \\
\end{array}$ \\
\hline $\begin{array}{c}\text { B-8;Li-8; } \\
\text { Na-8;Si-76 } \\
\text { (Frit 418) }^{\dagger \dagger}\end{array}$ & $\begin{array}{c}- \\
25-41 \\
\mathrm{~T}_{\mathrm{L}} \\
44 \\
\end{array}$ & $\begin{array}{c}- \\
25-43 \\
\mathrm{~T}_{\mathrm{L}}, \mathrm{Neph} \\
44 \\
\end{array}$ \\
\hline $\begin{array}{l}\text { B-10;Li-9; } \\
\text { Na-8;Si-73 }\end{array}$ & $\begin{array}{c}- \\
25-42 \\
\mathrm{~T}_{\mathrm{L}}, \mathrm{Neph} \\
43\end{array}$ & $\begin{array}{c}- \\
25-42 \\
\text { Neph } \\
43\end{array}$ \\
\hline $\begin{array}{l}\text { B-14;Li-8; } \\
\text { Na-8;Si-70 }\end{array}$ & $\begin{array}{c}- \\
25-41 \\
\mathrm{~T}_{\mathrm{L}}, \text { Neph } \\
42\end{array}$ & $\begin{array}{c}- \\
25-41 \\
\text { Neph } \\
42 \\
\end{array}$ \\
\hline $\begin{array}{l}\text { B-8;Ca-3;Li-8; } \\
\text { Na-8;Si-73 }\end{array}$ & $\begin{array}{c}-\overline{-} \\
25-41 \\
\mathrm{~T}_{\mathrm{L}} \\
43\end{array}$ & $\begin{array}{c}- \\
25-42 \\
\text { Neph } \\
43\end{array}$ \\
\hline $\begin{array}{c}\text { B-10;Ca-3;Li-9; } \\
\text { Na-8;Si-70 }\end{array}$ & $\begin{array}{c}- \\
25-41 \\
\text { Neph } \\
42\end{array}$ & $\begin{array}{c}- \\
25-41 \\
\text { Neph } \\
42\end{array}$ \\
\hline
\end{tabular}

Note that these frits are also included in Table 3-10.

${ }^{\dagger}$ Note that the results for Frit 418 are also highlighted in Table 3-9.

Sludge composition intervals were developed for a Variation Stage assessment encompassing the JRZ-04 composition projection, with and without ARP, as shown in Table 3-12, since the washing scenario considered in developing this composition projection was considered the most likely to be used (based on the improvement in projected operating windows with Frit 418). The EVs of this region were then identified and combined with the 13 frit compositions described in Table 3-10 and Table 3-11 over waste loadings of 25-50\%. Each glass composition was then evaluated against the PCCS MAR, and the results are given in Table 3-13. 
Table 3-12. Sludge Composition Intervals Developed for the JRZ-04 Composition Projection.

\begin{tabular}{|c|c|c|c|c|c|c|}
\hline Oxide & $\mathbf{A l}_{2} \mathbf{O}_{\mathbf{3}}$ & $\mathbf{C a O}$ & $\mathbf{F e}_{2} \mathbf{O}_{\mathbf{3}}$ & $\mathbf{M g O}$ & $\mathbf{M n O}$ & $\mathbf{N a}_{\mathbf{2}} \mathbf{O}$ \\
\hline Minimum (wt \%) & 25.28 & 1.44 & 24.28 & 0.21 & 6.66 & 19.04 \\
\hline Maximum (wt \%) & 30.55 & 2.49 & 29.00 & 1.24 & 7.88 & 23.16 \\
\hline \multicolumn{7}{|l|}{} \\
\hline Oxide & $\mathbf{N i O}$ & $\mathbf{S O}_{\mathbf{4}}{ }^{2-}$ & $\mathbf{S i O}_{2}$ & $\mathbf{T i O}_{\mathbf{2}}$ & $\mathbf{U}_{\mathbf{3}} \mathbf{O}_{\mathbf{8}}$ & Others \\
\hline Minimum (wt \%) & 2.78 & 0.91 & 1.96 & 0.00 & 4.70 & 0.77 \\
\hline Maximum (wt \%) & 3.88 & 1.50 & 3.05 & 1.82 & 5.80 & 1.80 \\
\hline
\end{tabular}

Table 3-13. Variation Stage MAR Assessment Results for the JRZ-04 Composition Projection.

\begin{tabular}{|c|c|c|c|c|c|}
\hline $\begin{array}{l}\text { Frit Oxides } \\
\text { (wt \%) }\end{array}$ & $\begin{array}{c}\text { B-10;Ca-3; } \\
\text { Li-9;Na-8;Si-70 }\end{array}$ & $\begin{array}{l}\text { B-10;Li-11; } \\
\text { Na-6;Si-73 }\end{array}$ & $\begin{array}{l}\text { B-10;Li-9; } \\
\text { Na-8;Si-73 }\end{array}$ & $\begin{array}{c}\text { B-12;Ca-3; } \\
\text { Li-9;Na-6; } \\
\text { Si-70 } \\
\text { (Frit IS1)* }\end{array}$ & $\begin{array}{l}\text { B-12;Li-9; } \\
\text { Na-8;Si-71 }\end{array}$ \\
\hline $\begin{array}{c}\text { Variation } \\
\text { Stage MAR } \\
\text { Outcome }\end{array}$ & $\begin{array}{c}- \\
25-37 \\
\text { lowv, } \mathrm{T}_{\mathrm{L}} \\
39 \\
\end{array}$ & $\begin{array}{c}- \\
25-36 \\
\text { lowv } \\
42 \\
\end{array}$ & $\begin{array}{c}- \\
25-37 \\
\mathrm{~T}_{\mathrm{L}} \\
40 \\
\end{array}$ & $\begin{array}{c}- \\
25-35 \\
\mathrm{~T}_{\mathrm{L}} \\
41 \\
\end{array}$ & $\begin{array}{c}- \\
25-36 \\
\text { lowv } \\
40 \\
\end{array}$ \\
\hline $\begin{array}{l}\text { Frit Oxides } \\
\text { (wt \%) }\end{array}$ & $\begin{array}{l}\text { B-14;Li-8; } \\
\text { Na-8;Si-70 }\end{array}$ & $\begin{array}{l}\text { B-14;Li-9; } \\
\text { Na-6;Si-71 } \\
\text { (Frit IS2)* }\end{array}$ & $\begin{array}{c}\text { B-8;Ca-3; } \\
\text { Li-10;Na-8; } \\
\text { Si-71 }\end{array}$ & $\begin{array}{c}\text { B-8;Ca-3; } \\
\text { Li-11;Na-6; } \\
\text { Si-72 } \\
\text { (Frit IS3)* }\end{array}$ & $\begin{array}{c}\text { B-8;Ca-3; } \\
\text { Li-8;Na-8; } \\
\text { Si-73 }\end{array}$ \\
\hline $\begin{array}{c}\text { Variation } \\
\text { Stage MAR } \\
\text { Outcome }\end{array}$ & $\begin{array}{c}25-36 \\
\mathrm{~T}_{\mathrm{L}} \\
39 \\
\end{array}$ & $\begin{array}{c}25-35 \\
\mathrm{~T}_{\mathrm{L}} \\
41 \\
\end{array}$ & $\begin{array}{c}25-36 \\
\text { lowv } \\
40 \\
\end{array}$ & $\begin{array}{c}- \\
25-37 \\
\text { lowv, } \mathrm{T}_{\mathrm{L}} \\
41\end{array}$ & $\begin{array}{c}25-36 \\
\mathrm{~T}_{\mathrm{L}} \\
40 \\
\end{array}$ \\
\hline $\begin{array}{l}\text { Frit Oxides } \\
(\text { wt \%) }\end{array}$ & $\begin{array}{l}\text { B-8;Li-10; } \\
\text { Na-8;Si-74 }\end{array}$ & $\begin{array}{l}\text { B-8;Li-11; } \\
\text { Na-6;Si-75 } \\
\text { (Frit IS4)* }\end{array}$ & $\begin{array}{c}\text { B-8;Li-8; } \\
\text { Na-8;Si-76 } \\
\text { (Frit 418)* }\end{array}$ & & \\
\hline $\begin{array}{c}\text { Variation } \\
\text { Stage MAR } \\
\text { Outcome }\end{array}$ & $\begin{array}{c}- \\
25-38 \\
\mathrm{~T}_{\mathrm{L}} \\
41 \\
\end{array}$ & $\begin{array}{c}- \\
25-37 \\
\mathrm{~T}_{\mathrm{L}} \\
43 \\
\end{array}$ & $\begin{array}{c}\text { highv } \\
28-37 \\
\mathrm{~T}_{\mathrm{L}} \\
42 \\
\end{array}$ & & \\
\hline
\end{tabular}

In general, the Variation Stage results show that the projected operating windows where all the EVs are predicted to produce acceptable glasses are narrower than the Nominal Stage projections. The systems are limited by liquidus temperature or low viscosity predictions at waste loadings around $36 \%$. Nepheline crystallization is predicted for some EVs around $40 \% \mathrm{WL}$.

Schedule constraints dictated that frit fabrication to support melt rate testing begin at this point in the frit optimization process. Therefore, five of these candidate frit compositions were selected for use in melt rate testing based on the Nominal and Variation Stage MAR assessment results. Those frits marked with an asterisk (*) in Table 3-13 were selected for use in melt rate testing and given identifiers. In addition to the MAR assessment results, these frits were also selected to help identify the impacts of $\mathrm{B}_{2} \mathrm{O}_{3}$ concentration (e.g., 8 wt \% $\mathrm{B}_{2} \mathrm{O}_{3}$ in Frit 418 versus 14 wt $\% \mathrm{~B}_{2} \mathrm{O}_{3}$ in 
Frit IS2), $\mathrm{CaO}$ concentration (e.g., 0 wt \% $\mathrm{CaO}$ in Frit IS4 versus 3 wt \% $\mathrm{CaO}$ in Frit IS3), and $\mathrm{Li}_{2} \mathrm{O}$ concentration (e.g., 8 wt $\% \mathrm{Li}_{2} \mathrm{O}$ in Frit 418 versus 11 wt $\% \mathrm{Li}_{2} \mathrm{O}$ in Frit IS4) on melt rate.

\subsection{Mid-December 2009 SB6 Composition Projections}

SRNL developed composition projections for the Tank 51 SB6 batch after washing (both a baseline washing scenario, JRZ-03, and a scenario with one less wash, JRZ-04) and the Tank 40 Sludge Batch 5 (SB5) heel prior to blending. These individual tank compositions were used to project various blending scenarios to gauge the impact of blend point on potential frit performance. The baseline blend point was approximately $75 \mathrm{wt} \%$ Tank 51 and $25 \mathrm{wt} \%$ Tank 40 to constitute the SB6 blend. SRNL also projected blend points of 85/15 and 65/35 (Tank $51 /$ Tank 40 , wt \%) using a simple rule of mixtures approach to bound potential variation in the blend point. The impact of ARP additions was not considered in this portion of the evaluation. The resulting composition projections are given in Table 3-14. 
Table 3-14. SRNL Composition Projections for Various SB6 Blend Points.

\begin{tabular}{|c|c|c|c|c|c|c|}
\hline Scenario & \multicolumn{2}{|c|}{ Baseline } & \multicolumn{2}{|c|}{ More SB5 Used } & \multicolumn{2}{|c|}{ Less SB5 Used } \\
\hline $\begin{array}{c}\text { Sludge Composition } \\
\text { Projection }\end{array}$ & JRZ-03 & JRZ-04 & JRZ-03 & JRZ-04 & JRZ-03 & JRZ-04 \\
\hline $\begin{array}{l}\text { Fraction of Tank } 40 \\
\text { in blend }\end{array}$ & 0.27 & 0.26 & 0.15 & 0.15 & 0.35 & 0.35 \\
\hline $\begin{array}{c}\text { Fraction of Tank } 51 \\
\text { in blend }\end{array}$ & 0.73 & 0.74 & 0.85 & 0.85 & 0.65 & 0.65 \\
\hline $\begin{array}{c}\text { Tank } 40 \text { Mass Prior } \\
\text { to Transfer }(\mathrm{kg})\end{array}$ & 56,123 & 56,123 & 27,269 & 28,207 & 83,204 & 86,066 \\
\hline $\begin{array}{c}\text { Tank } 51 \text { Transferred } \\
\text { Mass }(\mathrm{kg})\end{array}$ & 154,523 & 159,838 & 154,523 & 159,838 & 154,523 & 159,838 \\
\hline $\mathrm{Al}_{2} \mathrm{O}_{3}$ & 29.03 & 28.49 & 30.14 & 29.45 & 28.24 & 27.70 \\
\hline $\mathrm{B}_{2} \mathrm{O}_{3}$ & 0.01 & 0.01 & 0.01 & 0.01 & 0.01 & 0.01 \\
\hline $\mathrm{BaO}$ & 0.15 & 0.15 & 0.15 & 0.15 & 0.15 & 0.14 \\
\hline $\mathrm{CaO}$ & 1.80 & 1.76 & 1.70 & 1.65 & 1.88 & 1.85 \\
\hline $\mathrm{CdO}$ & 0.12 & 0.11 & 0.12 & 0.12 & 0.11 & 0.11 \\
\hline $\mathrm{Ce}_{2} \mathrm{O}_{3}$ & 0.26 & 0.25 & 0.27 & 0.26 & 0.25 & 0.24 \\
\hline $\mathrm{Cr}_{2} \mathrm{O}_{3}$ & 0.16 & 0.16 & 0.15 & 0.15 & 0.16 & 0.16 \\
\hline $\mathrm{CuO}$ & 0.13 & 0.12 & 0.13 & 0.13 & 0.12 & 0.12 \\
\hline $\mathrm{Fe}_{2} \mathrm{O}_{3}$ & 27.72 & 27.04 & 27.68 & 26.89 & 27.75 & 27.15 \\
\hline $\mathrm{Gd}_{2} \mathrm{O}_{3}$ & 0.06 & 0.05 & 0.05 & 0.05 & 0.06 & 0.06 \\
\hline $\mathrm{K}_{2} \mathrm{O}$ & 0.06 & 0.07 & 0.06 & 0.07 & 0.06 & 0.06 \\
\hline $\mathrm{La}_{2} \mathrm{O}_{3}$ & 0.11 & 0.11 & 0.12 & 0.11 & 0.11 & 0.11 \\
\hline $\mathrm{MgO}$ & 0.76 & 0.74 & 0.63 & 0.61 & 0.86 & 0.85 \\
\hline $\mathrm{MnO}$ & 7.54 & 7.36 & 7.82 & 7.59 & 7.35 & 7.17 \\
\hline $\mathrm{Na}_{2} \mathrm{O}$ & 18.94 & 20.63 & 18.05 & 20.05 & 19.58 & 21.10 \\
\hline $\mathrm{NiO}$ & 3.48 & 3.39 & 3.54 & 3.44 & 3.43 & 3.36 \\
\hline $\mathrm{PbO}$ & 0.04 & 0.03 & 0.04 & 0.03 & 0.04 & 0.04 \\
\hline $\mathrm{SO}_{4}{ }^{2-}$ & 1.06 & 1.16 & 1.07 & 1.19 & 1.06 & 1.15 \\
\hline $\mathrm{SiO}_{2}$ & 2.62 & 2.55 & 2.63 & 2.56 & 2.60 & 2.55 \\
\hline $\mathrm{SrO}$ & 0.08 & 0.07 & 0.08 & 0.08 & 0.08 & 0.07 \\
\hline $\mathrm{TiO}_{2}$ & 0.02 & 0.02 & 0.01 & 0.01 & 0.02 & 0.02 \\
\hline $\mathrm{U}_{3} \mathrm{O}_{8}$ & 5.44 & 5.31 & 5.08 & 4.95 & 5.70 & 5.60 \\
\hline $\mathrm{ZnO}$ & 0.07 & 0.07 & 0.07 & 0.06 & 0.07 & 0.07 \\
\hline $\mathrm{ZrO}_{2}$ & 0.35 & 0.34 & 0.40 & 0.38 & 0.32 & 0.31 \\
\hline
\end{tabular}

A Nominal Stage assessment was performed by combining the sludge compositions in Table 3-14 with the frit compositions described earlier in Table 3-3 over waste loadings of $25-60 \%$. The resulting glass compositions were evaluated against the PCCS MAR and projected operating windows were determined. The results showed that the impact of a change in blend point was minimal for the projected performance of these frits. For example, the projected operating windows for Frit 418 at the various blend points are described in Table 3-15. There is no practical difference in the projected operating windows or limiting constraints for Frit 418 at the three blend points. The results for the other frit compositions were similar, again indicating that there is little practical impact of the uncertainty in blend point on projected operating windows for candidate frits. 
SRNL-STI-2010-00137

Revision 0

Table 3-15. Nominal Stage MAR Assessment Results for Frit 418 at Various SB6 Blend Points.

\begin{tabular}{|c|c|c|c|}
\hline $\begin{array}{c}\text { Frit Oxides } \\
\text { (wt \%) }\end{array}$ & JRZ-04 & $\begin{array}{c}\text { JRZ-04 with } \\
\text { more SB5 used } \\
\text { (85/15 blend) }\end{array}$ & $\begin{array}{c}\text { JRZ-04 with less } \\
\text { SB5 used } \\
\text { (65/35 blend) }\end{array}$ \\
\hline B-8;Li-8; & - & - & - \\
Na-8;Si-76 & $25-41$ & $25-41$ & $25-42$ \\
(Frit 418) & $\mathrm{T}_{\mathrm{L}}$ & $\mathrm{T}_{\mathrm{L}}$ & $\mathrm{T}_{\mathrm{L}}$ \\
& 44 & 44 & 44 \\
\hline
\end{tabular}

\subsection{Late December 2009 SB6 Composition Projections}

SRR provided feedback that it may be necessary to transfer the SB6 batch material to Tank 40 prior to the completion of decanting in Tank 51. In this case, some of the blended material would need to be fed to DWPF for approximately six weeks prior to a planned melter outage at DWPF. Decanting of SB6 would then be completed in Tank 40 during the melter outage. SRNL developed composition projections for these scenarios, given in Table 3-16. The projection of the SB6 blend prior to the Tank 40 decant is identified as JRZ-05, and JRZ-06 represents the SB6 blend composition after a 265,000 gallon decant of Tank 40 . 
SRNL-STI-2010-00137

Revision 0

Table 3-16. SRNL SB6 Composition Projections Before and After a Decant in Tank 40.

\begin{tabular}{|c|c|c|}
\hline Oxide (wt \%) & JRZ-05 & JRZ-06 \\
\hline $\mathrm{Al}_{2} \mathrm{O}_{3}$ & 26.01 & 29.24 \\
\hline $\mathrm{BaO}$ & 0.13 & 0.15 \\
\hline $\mathrm{CaO}$ & 1.75 & 2.05 \\
\hline $\mathrm{CdO}$ & 0.10 & 0.12 \\
\hline $\mathrm{Ce}_{2} \mathrm{O}_{3}$ & 0.22 & 0.26 \\
\hline $\mathrm{Cr}_{2} \mathrm{O}_{3}$ & 0.15 & 0.16 \\
\hline $\mathrm{CuO}$ & 0.11 & 0.12 \\
\hline $\mathrm{Fe}_{2} \mathrm{O}_{3}$ & 23.85 & 27.90 \\
\hline $\mathrm{Gd}_{2} \mathrm{O}_{3}$ & 0.05 & 0.05 \\
\hline $\mathrm{K}_{2} \mathrm{O}$ & 0.09 & 0.06 \\
\hline $\mathrm{La}_{2} \mathrm{O}_{3}$ & 0.10 & 0.11 \\
\hline $\mathrm{MgO}$ & 0.66 & 0.77 \\
\hline $\mathrm{MnO}$ & 6.49 & 7.59 \\
\hline $\mathrm{Na}_{2} \mathrm{O}$ & 28.29 & 18.20 \\
\hline $\mathrm{NiO}$ & 2.99 & 3.50 \\
\hline $\mathrm{PbO}$ & 0.03 & 0.03 \\
\hline $\mathrm{SO}_{4}{ }^{2-}$ & 1.61 & 1.08 \\
\hline $\mathrm{SiO}_{2}$ & 2.25 & 2.61 \\
\hline $\mathrm{SrO}$ & 0.07 & 0.08 \\
\hline $\mathrm{TiO}_{2}$ & 0.01 & 0.02 \\
\hline $\mathrm{U}_{3} \mathrm{O}_{8}$ & 4.68 & 5.48 \\
\hline $\mathrm{ZnO}$ & 0.06 & 0.07 \\
\hline $\mathrm{ZrO}_{2}$ & 0.30 & 0.36 \\
\hline $\begin{array}{c}\text { Supernate } \mathrm{Na}^{+} \\
(\mathrm{M})\end{array}$ & 0.870 & 0.870 \\
\hline $\begin{array}{c}\text { Insoluble } \\
\text { solids (wt \%) }\end{array}$ & 7.01 & 12.05 \\
\hline
\end{tabular}

A Nominal Stage MAR assessment was performed using the frit composition array described earlier in Table 3-3 and the two sludge compositions given in Table 3-16. The results were screened to identify candidate frits that provided projected operating windows of at least $28-40 \%$ WL with each of the projections, with 1148 frit compositions meeting this criterion for JRZ-05 and 175 for JRZ-06. The Nominal Stage results for Frit 418 are shown in Table 3-17. The projected operating window for JRZ-05 with Frit 418 is nepheline limited at $40 \%$ waste loading. Nepheline predictions move up to $45 \%$ waste loading with JRZ-06, but liquidus temperature predictions limit the projected operating window to $38 \%$ waste loading.

Two additional frits of interest for melt rate testing were also down-selected from the Nominal Stage assessment results for projection JRZ-06. The Nominal Stage results for these two frits (given identifiers IS5 and IS6) are included in Table 3-17. Note that these frits are not viable for use with the JRZ-05 projection. The frits were selected since their projected operating windows were larger than that for Frit 418 with JRZ-06, and also to evaluate the impact of a higher alkali concentration (17 and $19 \mathrm{wt} \% \mathrm{Li}_{2} \mathrm{O}+\mathrm{Na}_{2} \mathrm{O}$ for Frits IS5 and IS6, respectively) on melt rate for SB6. 
Table 3-17. Nominal Stage MAR Assessment Results for Frit 418 with Projections JRZ-05 and JRZ-06.

\begin{tabular}{|c|c|c|}
\hline $\begin{array}{c}\text { Frit Oxides } \\
\text { (wt \%) }\end{array}$ & JRZ-05 & JRZ-06 \\
\hline B-8;Li-8; & - & - \\
Na-8;Si-76 & $25-40$ & $25-38$ \\
(Frit 418) & Neph & $\mathrm{T}_{\mathrm{L}}$ \\
\hline B-10;Li-12; & 41 & 45 \\
Na-6;Si-72 & $25-27$ & - \\
(IS5) & lowv & $25-41$ \\
\hline B-8;Li-11; & - & $\mathrm{T}_{\mathrm{L}}$ \\
Na-8;Si-73 & $25-31$ & 44 \\
(IS6) & lowv & $25-40$ \\
\hline
\end{tabular}

\subsection{Early January 2010 SB6 Composition Projections}

A revised washing spreadsheet was received via email from SRR on December 21, 2009. The changes accounted for the reduction in the number of SB6 washes as recommended by SRNL to increase the sludge $\mathrm{Na}_{2} \mathrm{O}$ concentration, as well as analyses of the SB6 qualification sample in the SRNL shielded cells. SRNL developed new SB6 composition projections based on this revised washing strategy, which are given in Table 3-18. Composition JRZ-07 represents the SB6 blend in Tank 40 prior to the decant, and JRZ-08 represents the Tank 40 composition after the decant. Composition JRZ-09 is an average of each of the components of JRZ-07 and JRZ-08 to provide an estimate for a scenario where only one-half of the planned decant volume is removed. 
Table 3-18. SB6 Composition Projections Before and After a Tank 40 Decant Using a Revised Washing Strategy.

\begin{tabular}{|c|c|c|c|}
\hline Oxide (wt \%) & JRZ-07 & JRZ-08 & JRZ-09 \\
\hline $\mathrm{Al}_{2} \mathrm{O}_{3}$ & 26.69 & 28.94 & 27.81 \\
\hline $\mathrm{BaO}$ & 0.13 & 0.15 & 0.14 \\
\hline $\mathrm{CaO}$ & 1.82 & 2.02 & 1.92 \\
\hline $\mathrm{CdO}$ & 0.10 & 0.12 & 0.11 \\
\hline $\mathrm{Ce}_{2} \mathrm{O}_{3}$ & 0.23 & 0.26 & 0.24 \\
\hline $\mathrm{Cr}_{2} \mathrm{O}_{3}$ & 0.15 & 0.16 & 0.16 \\
\hline $\mathrm{CuO}$ & 0.11 & 0.12 & 0.12 \\
\hline $\mathrm{Fe}_{2} \mathrm{O}_{3}$ & 24.72 & 27.49 & 26.10 \\
\hline $\mathrm{Gd}_{2} \mathrm{O}_{3}$ & 0.05 & 0.05 & 0.05 \\
\hline $\mathrm{K}_{2} \mathrm{O}$ & 0.09 & 0.06 & 0.07 \\
\hline $\mathrm{La}_{2} \mathrm{O}_{3}$ & 0.10 & 0.11 & 0.10 \\
\hline $\mathrm{MgO}_{\mathrm{MnO}}$ & 0.68 & 0.76 & 0.72 \\
\hline $\mathrm{Na}_{2} \mathrm{O}$ & 26.72 & 7.47 & 7.10 \\
\hline $\mathrm{NiO}_{\mathrm{PbO}}$ & 3.10 & 19.29 & 22.78 \\
\hline $\mathrm{SO}_{4}{ }^{2-}$ & 0.03 & 0.03 & 3.28 \\
\hline $\mathrm{SiO}_{2}$ & 2.36 & 1.03 & 0.03 \\
\hline $\mathrm{SrO}_{\mathrm{TiO}}$ & 0.07 & 0.08 & 0.19 \\
\hline $\mathrm{U}_{3} \mathrm{O}_{8}$ & 0.01 & 0.02 & 0.02 \\
\hline $\mathrm{ZnO}$ & 4.86 & 5.40 & 5.13 \\
\hline $\mathrm{ZrO}_{2}$ & 0.06 & 0.07 & 0.06 \\
\hline $\begin{array}{c}\mathrm{Supernate} \\
(\mathrm{Ma})\end{array}$ & 0.966 & 0.966 & 0.966 \\
\hline $\begin{array}{c}\mathrm{Insoluble}^{+} \\
\text {solids (wt \%) }\end{array}$ & 8.28 & 12.04 & 10.16 \\
\hline \hline
\end{tabular}

The impact of ARP additions was calculated, resulting in a total of six SB6 projections. Each of these sludge compositions was combined with a smaller array of 1,144 frit compositions over waste loadings of $25-60 \%$ for a Nominal Stage MAR assessment. $\mathrm{CaO}$ was removed from the frit array at this stage since it was no longer seen to be necessary for improved sulfate retention for SB6. Table 3-19 provides the minimum and maximum concentrations of each of the frit components in the smaller array. The Nominal Stage results for each of the SB6 composition projections with Frit 418 are given in Table 3-20.

Table 3-19. Array of Frit Compositions for Nominal Stage Assessments.

\begin{tabular}{|c|c|c|c|c|}
\hline & $\mathbf{B}_{2} \mathbf{O}_{3}$ & $\mathbf{L i}_{2} \mathbf{O}$ & $\mathbf{N a}_{2} \mathbf{O}$ & $\mathbf{S i O}_{2}$ \\
\hline $\begin{array}{c}\text { Minimum } \\
(\mathrm{wt} \%)\end{array}$ & 8.0 & 5.0 & 2.0 & 56.0 \\
\hline $\begin{array}{c}\text { Maximum } \\
(\mathrm{wt} \%)\end{array}$ & 20.0 & 12.0 & 12.0 & 85.0 \\
\hline
\end{tabular}


SRNL-STI-2010-00137

Revision 0

Table 3-20. Nominal Stage MAR Assessment Results for Frit 418 with the Early January 2010 SB6 Composition Projections.

\begin{tabular}{|c|c|c|c|}
\hline $\begin{array}{c}\text { Frit Oxides } \\
\text { (wt \%) }\end{array}$ & JRZ-07 & JRZ-07ARP & JRZ-08 \\
\hline $\begin{array}{c}\text { B-8;Li-8; } \\
\text { Na-8;Si-76 } \\
\text { (Frit 418) }\end{array}$ & $\begin{array}{c}- \\
25-41 \\
\text { Neph } \\
42\end{array}$ & $\begin{array}{c}- \\
25-41 \\
\text { Neph } \\
42\end{array}$ & $\begin{array}{c}- \\
25-40 \\
\mathrm{~T}_{\mathrm{L}} \\
44\end{array}$ \\
\hline & JRZ-08ARP & JRZ-09 & JRZ-09ARP \\
\hline $\begin{array}{c}\text { B-8;Li-8; } \\
\text { Na-8;Si-76 } \\
\text { (Frit 418) }\end{array}$ & $\begin{array}{c}- \\
25-42 \\
\mathrm{~T}_{\mathrm{L}} \\
45\end{array}$ & $\begin{array}{c}- \\
25-42 \\
\text { Neph } \\
43\end{array}$ & $\begin{array}{c}- \\
25-42 \\
\text { Neph } \\
43\end{array}$ \\
\hline
\end{tabular}

Each system with Frit 418 is limited by either nepheline crystallization or liquidus temperature predictions at $40-42 \% \mathrm{WL}$. The projected operating windows would be further reduced if expected variation in the sludge compositions was taken into account. DWPF plans to target a waste loading of $36 \%$ for SB6 processing. While it would be beneficial from a plant operating perspective to continue using Frit 418 for SB6 vitrification - since it's currently used for SB5 alternative frits may provide wider projected operating windows, and would thus provide more of a buffer around the $36 \%$ WL target.

It would be desirable to utilize the same frit composition for SB6 both before and after the decant in Tank 40. That is, a single frit composition that provides a viable projected operating window for compositions JRZ-07, -08 and -09, with and without ARP, is of interest. A further review of the Nominal Stage results showed that there are 15 frit compositions available that can provide projected operating windows of at least $30-40 \%$ WL for all six of the composition projections. However, the maximum projected waste loading for an acceptable glass among these frits is $41 \%$. In other words, there would be no practical improvement as compared to the projected performance of Frit 418.

Next, to provide further insight into potential frits for these SB6 projections, frit compositions were identified that provided projected operating windows of at least $30-40 \%$ WL for each of the three projections, individually, both with and without ARP. The approximate number of frits available for each of the SB6 composition projections and the maximum projected WL available are listed in Table 3-21. Higher waste loadings for each of the composition projections are achievable on an individual basis. Note the reduction in the number of available frits and maximum projected waste loading achievable for composition JRZ-08, which is likely due to its reduced $\mathrm{Na}_{2} \mathrm{O}$ concentration as compared to either JRZ-07 or JRZ-09. 
Table 3-21. Summary of Nominal Stage Assessment Results for Individual SB6 Composition Projections.

\begin{tabular}{|c|c|c|}
\hline $\begin{array}{c}\text { SB6 Composition } \\
\text { Projection }\end{array}$ & $\begin{array}{c}\text { Approximate Number of Frit } \\
\text { Compositions Providing a } \\
\text { Projected Operating Window of } \\
\text { At Least 30-40\%WL }\end{array}$ & $\begin{array}{c}\text { Maximum Projected } \\
\text { Waste Loading for } \\
\text { Acceptable Glass }\end{array}$ \\
\hline $\begin{array}{c}\text { JRZ-07 with and } \\
\text { without ARP }\end{array}$ & 275 & 46 \\
\hline $\begin{array}{c}\text { JRZ-08 with and } \\
\text { without ARP }\end{array}$ & 84 & 43 \\
\hline $\begin{array}{c}\text { JRZ-09 with and } \\
\text { without ARP }\end{array}$ & 236 & 45 \\
\hline
\end{tabular}

Based on the Nominal Stage MAR assessment results for the individual SB6 composition projections, seven frits, listed in Table 3-22, were down-selected to support Variation Stage MAR assessments. The composition projections for which each frit was selected are identified in the table. Frit 418 was again included, along with Frit 503, which was previously of interest for SB4 vitrification.

Table 3-22. Frit Compositions (wt \%) for a Variation Stage Assessment with SB6 Composition Projections JRZ-07, JRZ-08 and JRZ-09.

\begin{tabular}{||c|c|c|c|c||}
\hline $\begin{array}{c}\text { Relevant SB6 } \\
\text { Composition } \\
\text { Projections }\end{array}$ & $\mathbf{B}_{\mathbf{2}} \mathbf{O}_{\mathbf{3}}$ & $\mathbf{L i}_{\mathbf{2}} \mathbf{O}$ & $\mathbf{N a}_{\mathbf{2}} \mathbf{O}$ & $\mathbf{S i O}_{\mathbf{2}}$ \\
\hline $\begin{array}{c}\text { JRZ-08, } \\
\text { JRZ-08ARP }\end{array}$ & 10 & 11 & 7 & 72 \\
\cline { 2 - 5 } & 11 & 9 & 9 & 71 \\
\hline $\begin{array}{c}\text { JRZ-08, } \\
\text { JRZ-08ARP, } \\
\text { JRZ-09, } \\
\text { JRZ-09ARP }\end{array}$ & 8 & 10 & 7 & 75 \\
\cline { 2 - 5 } & 8 & 10 & 8 & 74 \\
\hline $\begin{array}{c}\text { All } \\
\text { (Frit 418) }\end{array}$ & 8 & 8 & 8 & 76 \\
\hline $\begin{array}{c}\text { JRZ-07, } \\
\text { JRZ-07ARP, } \\
\text { JRZ-09, } \\
\text { JRZ-09ARP } \\
\text { (Frit 503) }\end{array}$ & 14 & 8 & 4 & 74 \\
\hline
\end{tabular}

The Nominal Stage results for these frits with the relevant SB6 composition projections are given in Table 3-23. The projected operating windows for these systems continue to be limited to around $41-42 \%$ WL. Since no practical improvement seemed to be available even when the sludge compositions were considered individually, a series of Variation Stage assessments was next performed to determine whether a target of 36\% WL for SB6 would be reasonable for any candidate frit composition. 
Table 3-23. Nominal Stage MAR Assessment Results for Selected Frits Combined with Projections JRZ-07, -08 and -09, with and without ARP.

\begin{tabular}{|c|c|c|c|c|c|c|}
\hline $\begin{array}{c}\text { Frit Oxides } \\
(\text { wt \%) }\end{array}$ & JRZ-08 & JRZ-08ARP & JRZ-09 & JRZ-09ARP & JRZ-07 & JRZ-07ARP \\
\hline $\begin{array}{l}\text { B-10;Li-11; } \\
\text { Na-7;Si-72 }\end{array}$ & $\begin{array}{c}- \\
25-41 \\
\mathrm{~T}_{\mathrm{L}} \\
45\end{array}$ & $\begin{array}{c}- \\
25-41 \\
\text { lowv } \\
44\end{array}$ & - & - & - & - \\
\hline $\begin{array}{l}\text { B-11;Li-9; } \\
\text { Na-9;Si-71 }\end{array}$ & $\begin{array}{c}- \\
25-41 \\
\mathrm{~T}_{\mathrm{L}}, \text { Neph } \\
42 \\
\end{array}$ & $\begin{array}{c}- \\
25-41 \\
\text { Neph } \\
42 \\
\end{array}$ & - & - & - & - \\
\hline $\begin{array}{l}\text { B-8;Li-10; } \\
\text { Na-7;Si-75 }\end{array}$ & $\begin{array}{c}- \\
25-41 \\
\mathrm{~T}_{\mathrm{L}} \\
45 \\
\end{array}$ & $\begin{array}{c}- \\
25-42 \\
\mathrm{~T}_{\mathrm{L}} \\
45 \\
\end{array}$ & $\begin{array}{c}- \\
25-43 \\
\text { Neph } \\
44 \\
\end{array}$ & $\begin{array}{c}- \\
25-43 \\
\text { Neph } \\
44 \\
\end{array}$ & - & - \\
\hline $\begin{array}{l}\text { B-8;Li-10; } \\
\text { Na-8;Si-74 }\end{array}$ & $\begin{array}{c}- \\
25-41 \\
\mathrm{~T}_{\mathrm{L}} \\
44 \\
\end{array}$ & $\begin{array}{c}- \\
25-43 \\
\mathrm{~T}_{\mathrm{L}}, \mathrm{Neph} \\
44\end{array}$ & $\begin{array}{c}- \\
25-41 \\
\text { Neph } \\
42 \\
\end{array}$ & $\begin{array}{c}- \\
25-42 \\
\text { lowv, Neph } \\
43 \\
\end{array}$ & - & - \\
\hline $\begin{array}{l}\text { B-8;Li-11; } \\
\text { Na-7;Si-74 }\end{array}$ & $\begin{array}{c}- \\
25-41 \\
\mathrm{~T}_{\mathrm{L}} \\
44 \\
\end{array}$ & $\begin{array}{c}- \\
25-43 \\
\mathrm{~T}_{\mathrm{L}}, \mathrm{Neph} \\
44\end{array}$ & $\begin{array}{c}- \\
25-42 \\
\text { lowv, Neph } \\
43 \\
\end{array}$ & $\begin{array}{c}- \\
25-40 \\
\text { lowv } \\
43 \\
\end{array}$ & - & - \\
\hline $\begin{array}{c}\text { B-8;Li-8; } \\
\text { Na-8;Si-76 } \\
\text { (Frit 418) }\end{array}$ & $\begin{array}{c}- \\
25-40 \\
\mathrm{~T}_{\mathrm{L}} \\
44\end{array}$ & $\begin{array}{c}- \\
25-42 \\
\mathrm{~T}_{\mathrm{L}} \\
45\end{array}$ & $\begin{array}{c}- \\
25-42 \\
\text { Neph } \\
43\end{array}$ & $\begin{array}{c}- \\
25-42 \\
\text { Neph } \\
43\end{array}$ & $\begin{array}{c}- \\
25-41 \\
\text { Neph } \\
42\end{array}$ & $\begin{array}{c}- \\
25-41 \\
\text { Neph } \\
42\end{array}$ \\
\hline $\begin{array}{c}\text { B-14;Li-8; } \\
\text { Na-4;Si-74 } \\
\text { (Frit 503) }\end{array}$ & - & - & $\begin{array}{c}- \\
25-39 \\
\mathrm{~T}_{\mathrm{L}} \\
45\end{array}$ & $\begin{array}{c}- \\
25-41 \\
\mathrm{~T}_{\mathrm{L}} \\
45\end{array}$ & $\begin{array}{c}- \\
25-43 \\
\text { Neph } \\
44\end{array}$ & $\begin{array}{c}- \\
25-43 \\
\text { Neph } \\
44\end{array}$ \\
\hline
\end{tabular}

Frit/sludge combinations where only a "--" appears were not evaluated.

Sludge composition intervals were developed for Variation Stage assessments encompassing the three composition projections, both with and without ARP, as shown in Table 3-24. JRZ-09 was combined with JRZ-08 to further accommodate uncertainty in the Tank 40 decant volume. The EVs of this region were then identified and combined with the corresponding frit compositions in Table 3-22 over waste loadings of $25-50 \%$. Each resulting glass composition was then evaluated against the PCCS MAR, and the results are given in Table 3-25. 
Table 3-24. Sludge Composition Intervals for JRZ-07, -08 and -09, with and without ARP.

\begin{tabular}{|c|c|c|c|c|c|c||}
\hline \multirow{2}{*}{$\begin{array}{c}\text { Oxide } \\
\text { (wt \%) }\end{array}$} & \multicolumn{2}{|c|}{ JRZ-07 } & \multicolumn{2}{c|}{ JRZ-08 } & \multicolumn{2}{c|}{ JRZ-08 and JRZ-09 } \\
\cline { 2 - 7 } & Minimum & Maximum & Minimum & Maximum & Minimum & Maximum \\
\hline $\mathrm{Al}_{2} \mathrm{O}_{3}$ & 23.75 & 28.69 & 25.73 & 31.11 & 24.74 & 31.11 \\
\hline $\mathrm{CaO}$ & 1.28 & 2.32 & 1.47 & 2.52 & 1.38 & 2.52 \\
\hline $\mathrm{Fe}_{2} \mathrm{O}_{3}$ & 22.29 & 26.57 & 24.73 & 29.56 & 23.51 & 29.56 \\
\hline $\mathrm{MgO}$ & 0.15 & 1.18 & 0.23 & 1.26 & 0.19 & 1.26 \\
\hline $\mathrm{MnO}$ & 6.12 & 7.22 & 6.78 & 8.04 & 6.45 & 8.04 \\
\hline $\mathrm{Na}_{2} \mathrm{O}$ & 24.30 & 28.98 & 17.84 & 21.84 & 17.84 & 25.41 \\
\hline $\mathrm{NiO}$ & 2.52 & 3.60 & 2.85 & 3.95 & 2.68 & 3.95 \\
\hline $\mathrm{SO}_{4}{ }_{4}^{2-}$ & 1.11 & 1.68 & 0.78 & 1.37 & 0.78 & 1.53 \\
\hline $\mathrm{SiO}_{2}$ & 1.75 & 2.83 & 1.99 & 3.08 & 1.87 & 3.08 \\
\hline $\mathrm{TiO}_{2}$ & 0.00 & 1.81 & 0.00 & 1.81 & 0.00 & 1.81 \\
\hline $\mathrm{U}_{3} \mathrm{O}_{8}$ & 4.28 & 5.36 & 4.80 & 5.90 & 4.54 & 5.90 \\
\hline $\mathrm{Others}_{2}$ & 0.70 & 1.73 & 0.79 & 1.82 & 0.75 & 1.82 \\
\hline
\end{tabular}

Table 3-25. Variation Stage Results For Selected Frits with JRZ-07, JRZ-08, and JRZ-08 and -09 Combined.

\begin{tabular}{|c|c|c|c|}
\hline $\begin{array}{c}\text { Frit Oxides } \\
(\text { wt \%) }\end{array}$ & JRZ-07 & JRZ-08 & $\begin{array}{c}\text { JRZ-08 and } \\
\text { JRZ-09 } \\
\end{array}$ \\
\hline $\begin{array}{l}\text { B-10;Li-11; } \\
\text { Na-7;Si-72 }\end{array}$ & - & $\begin{array}{c}- \\
25-34 \\
\text { lowv } \\
41\end{array}$ & - \\
\hline $\begin{array}{l}\text { B-11;Li-9; } \\
\text { Na-9;Si-71 }\end{array}$ & - & $\begin{array}{c}- \\
25-37 \\
\text { lowv, } \mathrm{T}_{\mathrm{L}} \\
39\end{array}$ & - \\
\hline $\begin{array}{l}\text { B-8;Li-10; } \\
\text { Na-7;Si-75 }\end{array}$ & - & $\begin{array}{c}- \\
25-36 \\
\mathrm{~T}_{\mathrm{L}} \\
42\end{array}$ & $\begin{array}{c}- \\
25-36 \\
\mathrm{~T}_{\mathrm{L}} \\
41\end{array}$ \\
\hline $\begin{array}{l}\text { B-8;Li-10; } \\
\text { Na-8;Si-74 }\end{array}$ & - & $\begin{array}{c}- \\
25-37 \\
\mathrm{~T}_{\mathrm{L}} \\
41 \\
\end{array}$ & $\begin{array}{c}- \\
25-36 \\
\text { lowv, } T_{\mathrm{L}} \\
40\end{array}$ \\
\hline $\begin{array}{l}\text { B-8;Li-11; } \\
\text { Na-7;Si-74 }\end{array}$ & - & $\begin{array}{c}- \\
25-37 \\
\mathrm{~T}_{\mathrm{L}} \\
42\end{array}$ & $\begin{array}{c}- \\
25-34 \\
\text { lowv } \\
40\end{array}$ \\
\hline $\begin{array}{c}\text { B-8;Li-8; } \\
\text { Na-8;Si-76 } \\
\text { (Frit 418) }\end{array}$ & $\begin{array}{c}- \\
25-39 \\
\text { Neph } \\
40\end{array}$ & $\begin{array}{c}\text { highv } \\
32-35 \\
T_{L} \\
42 \\
\end{array}$ & $\begin{array}{c}\text { highv } \\
33-35 \\
\mathrm{~T}_{\mathrm{L}} \\
40 \\
\end{array}$ \\
\hline $\begin{array}{c}\text { B-14;Li-8; } \\
\text { Na-4;Si-74 } \\
\text { (Frit 503) }\end{array}$ & $\begin{array}{c}- \\
25-38 \\
\mathrm{~T}_{\mathrm{L}} \\
42\end{array}$ & $\begin{array}{c}\text { highv } \\
\text { no window } \\
\mathrm{T}_{\mathrm{L}} \\
44\end{array}$ & $\begin{array}{c}\text { highv } \\
\text { no window } \\
\mathrm{T}_{\mathrm{L}} \\
42\end{array}$ \\
\hline
\end{tabular}

Frit/sludge combinations where only a "-" appears were not evaluated. 
The Variation Stage results in Table 3-25 show that the projected operating windows over which all of the sludge EVs produce acceptable glasses are significantly reduced as compared with the projected operating windows in the Nominal Stage results (Table 3-23), particularly for the JRZ-08 and JRZ-09 composition projections. It would be difficult to target 36\% WL for SB6 using these frits. The performance of Frit 503 is particularly poor when variation is considered; therefore it was not included in the remainder of the study.

Another set of candidate frits, with their compositions given in Table 3-26, was selected from the Nominal Stage results to try to find improved projected operating windows at the Variation Stage for JRZ-08 and JRZ-09. These frits have higher $\mathrm{Na}_{2} \mathrm{O}$ concentrations in exchange for $\mathrm{Li}_{2} \mathrm{O}$. Results of the Variation Stage assessments with these three frits are given in Table 3-27. The projected operating windows over which all of the sludge EVs produce acceptable glasses remain too small for a target waste loading of $36 \%$.

Table 3-26. Candidate Frit Compositions with higher $\mathrm{Na}_{2} \mathrm{O}$ Concentrations (wt \%).

\begin{tabular}{|c|c|c|c||}
\hline $\mathbf{B}_{\mathbf{2}} \mathbf{O}_{\mathbf{3}}$ & $\mathbf{L i}_{\mathbf{2}} \mathbf{O}$ & $\mathbf{N a}_{\mathbf{2}} \mathbf{O}$ & $\mathbf{S i O}_{\mathbf{2}}$ \\
\hline 10 & 7 & 10 & 73 \\
\hline 12 & 7 & 10 & 71 \\
\hline 8 & 6 & 11 & 75 \\
\hline
\end{tabular}

Table 3-27. Variation Stage Results for Higher $\mathrm{Na}_{2} \mathrm{O}$ Concentration Frits.

\begin{tabular}{||c|c|c||}
\hline $\begin{array}{c}\text { Frit Oxides } \\
\text { (wt \%) }\end{array}$ & JRZ-08 & $\begin{array}{c}\text { JRZ-08 and } \\
\text { JRZ-09 }\end{array}$ \\
\hline & - & - \\
B-10;Li-7; & $25-36$ & $25-36$ \\
Na-10;Si-73 & $\mathrm{T}_{\mathrm{L}}$ & $\mathrm{T}_{\mathrm{L}}$ \\
& 39 & 38 \\
\hline & - & - \\
B-12;Li-7; & $25-36$ & $25-36$ \\
Na-10;Si-71 & $\mathrm{T}_{\mathrm{L}}$ & $\mathrm{T}_{\mathrm{L}}, \mathrm{Neph}$ \\
& 39 & 37 \\
\hline & highv & highv \\
B-8;Li-6; & $36-37$ & no window \\
Na-11;Si-75 & $\mathrm{T}_{\mathrm{L}}$ & $\mathrm{T}_{\mathrm{L}}$ \\
& 40 & 38 \\
\hline
\end{tabular}

Since Frit 418 provided acceptable glasses over at least some range of waste loadings for JRZ-07, JRZ-08 and JRZ-09 (see Table 3-25), two additional frits with compositions similar to Frit 418 were selected to again look for improvement in the projected operating windows when variation is applied. The compositions of these frits are given in Table 3-28. Variation Stage assessment results for these two frits are given in Table 3-29. There is little or no improvement in the projected operating windows for these frit compositions relative to Frit 418. 
SRNL-STI-2010-00137

Revision 0

Table 3-28. Candidate Frit Compositions Similar to Frit 418 (wt \%).

\begin{tabular}{|c|c|c|c|}
\hline $\mathbf{B}_{\mathbf{2}} \mathbf{O}_{\mathbf{3}}$ & $\mathbf{L i}_{\mathbf{2}} \mathbf{O}$ & $\mathbf{N a}_{\mathbf{2}} \mathbf{O}$ & $\mathbf{S i O}_{\mathbf{2}}$ \\
\hline 8 & 9 & 10 & 74 \\
\hline 8 & 8 & 9 & 75 \\
\hline
\end{tabular}

Table 3-29. Variation Stage Results for Frits Similar to Frit 418.

\begin{tabular}{|c|c|c|c|}
\hline $\begin{array}{c}\text { Frit Oxides } \\
\text { (wt \%) }\end{array}$ & JRZ-07 & JRZ-08 & $\begin{array}{c}\text { JRZ-08 and } \\
\text { JRZ-09 }\end{array}$ \\
\hline & - & - & - \\
B-8;Li-8; & $25-35$ & $25-37$ & $25-37$ \\
Na-10;Si-74 & $\Delta \mathrm{Gp}$ & $\mathrm{T}_{\mathrm{L}}$ & $\mathrm{T}_{\mathrm{L}}, \mathrm{Neph}$ \\
& 38 & 40 & 38 \\
\hline & - & - & - \\
B-8;Li-8; & $25-38$ & $25-37$ & $25-36$ \\
Na-9;Si-75 & Neph & $\mathrm{T}_{\mathrm{L}}$ & $\mathrm{T}_{\mathrm{L}}$ \\
& 39 & 41 & 39 \\
\hline
\end{tabular}

Composition projection JRZ-07 - the SB6 composition prior to the decant in Tank 40 - appears to be the most challenging of the group at this stage of the evaluation. To look for potential improvements in the projected operating window for this sludge, Nominal and Variation Stage assessments were performed with JRZ-07 only (paired with and without ARP, see Table 3-24) using a series of low $\mathrm{Na}_{2} \mathrm{O}$ concentration frits given in Table 3-30. These frits are not candidates for projections JRZ-08 or JRZ-09, but may offer some insight into the processing of JRZ-07.

Table 3-30. Candidate Frits with Low $\mathrm{Na}_{2} \mathrm{O}$ Concentrations for JRZ-07 (wt \%).

\begin{tabular}{||c|c|c|c||}
\hline $\mathbf{B}_{\mathbf{2}} \mathbf{O}_{\mathbf{3}}$ & $\mathbf{L i}_{\mathbf{2}} \mathbf{O}$ & $\mathbf{N a}_{\mathbf{2}} \mathbf{O}$ & $\mathbf{S i O}_{\mathbf{2}}$ \\
\hline 10 & 10 & 2 & 78 \\
\hline 10 & 9 & 2 & 79 \\
\hline 11 & 9 & 2 & 78 \\
\hline 8 & 10 & 2 & 80 \\
\hline 8 & 11 & 2 & 79 \\
\hline 8 & 9 & 3 & 80 \\
\hline 9 & 10 & 2 & 79 \\
\hline 9 & 11 & 2 & 78 \\
\hline
\end{tabular}

The results of the Variation Stage assessments with these frits are shown in Table 3-31. Many of the systems are limited at low waste loadings by predictions of high viscosity, which is not surprising given the low $\mathrm{Na}_{2} \mathrm{O}$ concentrations in the frits. Two of the frits offer projected operating windows up to $40 \% \mathrm{WL}$ over which all of the sludge EVs produce acceptable glasses, with nepheline crystallization predictions pushed up to 44 or $45 \%$ WL. Therefore, there appears to be some advantage to using lower $\mathrm{Na}_{2} \mathrm{O}$ concentration frits with JRZ-07. 
Table 3-31. Variation Stage Results for Low $\mathrm{Na}_{2} \mathrm{O}$ Concentration Frits with JRZ-07.

\begin{tabular}{|c|c|c|c|}
\hline $\begin{array}{c}\text { Frit Oxides } \\
\text { (wt \%) }\end{array}$ & JRZ-07 & $\begin{array}{c}\text { Frit Oxides } \\
\text { (wt \%) }\end{array}$ & JRZ-07 \\
\hline $\begin{array}{l}\text { B-10;Li-10; } \\
\text { Na-2;Si-78 }\end{array}$ & $\begin{array}{c}\text { highv } \\
27-39 \\
\mathrm{~T}_{\mathrm{L}} \\
44\end{array}$ & $\begin{array}{l}\text { B-8;Li-11; } \\
\text { Na-2;Si-79 }\end{array}$ & $\begin{array}{c}- \\
25-40 \\
\mathrm{~T}_{\mathrm{L}} \\
45\end{array}$ \\
\hline $\begin{array}{l}\text { B-10;Li-9; } \\
\text { Na-2;Si-79 }\end{array}$ & $\begin{array}{c}\text { highv } \\
35-38 \\
\mathrm{~T}_{\mathrm{L}} \\
45\end{array}$ & $\begin{array}{c}\text { B-8;Li-9; } \\
\text { Na-3;Si-80 }\end{array}$ & $\begin{array}{c}\text { highv } \\
36-39 \\
\mathrm{~T}_{\mathrm{L}} \\
44\end{array}$ \\
\hline $\begin{array}{l}\text { B-11;Li-9; } \\
\text { Na-2;Si-78 }\end{array}$ & $\begin{array}{c}\text { highv } \\
32-38 \\
\mathrm{~T}_{\mathrm{L}} \\
44\end{array}$ & $\begin{array}{l}\text { B-9;Li-10; } \\
\text { Na-2;Si-79 }\end{array}$ & $\begin{array}{c}\text { highv } \\
30-39 \\
\mathrm{~T}_{\mathrm{L}} \\
45 \\
\end{array}$ \\
\hline $\begin{array}{l}\text { B-8;Li-10; } \\
\text { Na-2;Si-80 }\end{array}$ & $\begin{array}{c}\text { highv } \\
33-39 \\
\mathrm{~T}_{\mathrm{L}} \\
45\end{array}$ & $\begin{array}{l}\text { B-9;Li-11; } \\
\text { Na-2;Si-78 }\end{array}$ & $\begin{array}{c}- \\
25-40 \\
\mathrm{~T}_{\mathrm{L}} \\
44\end{array}$ \\
\hline
\end{tabular}

Next, a further attempt was made to identify frit compositions that could provide good projected operating windows for projections JRZ-07, JRZ-08 and JRZ-09, albeit on an individual basis. A series of frits with $\mathrm{B}_{2} \mathrm{O}_{3}$ concentrations up to $14 \mathrm{wt} \%$ was selected, as shown in Table 3-32. Variation Stage assessment results for these frits are given in Table 3-33. In summary, there is again little improvement in the projected operating windows over which all of the sludge EVs produce acceptable glasses.

Table 3-32. Higher $\mathrm{B}_{2} \mathrm{O}_{3}$ Concentration Frits for Variation Stage Assessments (wt \%).

\begin{tabular}{|c|c|c|c||}
\hline \hline $\mathbf{B}_{\mathbf{2}} \mathbf{O}_{\mathbf{3}}$ & $\mathbf{L i}_{\mathbf{2}} \mathbf{O}$ & $\mathbf{N a}_{\mathbf{2}} \mathbf{O}$ & $\mathbf{S i O}_{\mathbf{2}}$ \\
\hline 10 & 10 & 3 & 77 \\
\hline 12 & 8 & 8 & 72 \\
\hline 12 & 8 & 9 & 71 \\
\hline 12 & 9 & 3 & 76 \\
\hline 14 & 8 & 9 & 69 \\
\hline
\end{tabular}


Table 3-33. Variation Stage Assessment Results for Higher $\mathrm{B}_{2} \mathrm{O}_{3}$ Concentration Frits.

\begin{tabular}{|c|c|c|c|}
\hline $\begin{array}{c}\text { Frit Oxides } \\
(\text { wt \%) }\end{array}$ & JRZ-07 & JRZ-08 & $\begin{array}{c}\text { JRZ-08 and } \\
\text { JRZ-09 } \\
\end{array}$ \\
\hline $\begin{array}{c}\text { B-10;Li-10; } \\
\text { Na-3;Si-77 }\end{array}$ & $\begin{array}{c}- \\
25-40 \\
\mathrm{~T}_{\mathrm{L}} \\
43 \\
\end{array}$ & - & - \\
\hline $\begin{array}{l}\text { B-12;Li-8; } \\
\text { Na-8;Si-72 }\end{array}$ & - & - & $\begin{array}{c}- \\
25-35 \\
\mathrm{~T}_{\mathrm{L}} \\
39\end{array}$ \\
\hline $\begin{array}{l}\text { B-12;Li-8; } \\
\text { Na-9;Si-71 }\end{array}$ & - & $\begin{array}{c}- \\
25-36 \\
\mathrm{~T}_{\mathrm{L}} \\
39\end{array}$ & - \\
\hline $\begin{array}{l}\text { B-12;Li-9; } \\
\text { Na-3;Si-76 }\end{array}$ & $\begin{array}{c}- \\
25-39 \\
\mathrm{~T}_{\mathrm{L}} \\
43\end{array}$ & - & - \\
\hline $\begin{array}{l}\text { B-14;Li-8; } \\
\text { Na-9;Si-69 }\end{array}$ & - & $\begin{array}{c}- \\
25-36 \\
\text { lowv, } \mathrm{T}_{\mathrm{L}} \\
38 \\
\end{array}$ & - \\
\hline
\end{tabular}

The multiple iterations of frit composition development described above for projections JRZ-07, JRZ-08 and JRZ-09 show that these systems will be challenging to process at a target waste loading of $36 \%$. Of all the factors explored, sodium concentration in the sludge appears to be the most influential in terms of the performance of potential frit compositions and remains an important variable in SB6 preparation. A series of additional SB6 composition projections was developed to further evaluate the impact of sludge $\mathrm{Na}_{2} \mathrm{O}$ concentration on frit optimization and perhaps provide guidance on washing of SB6. These projections are given in Table 3-34. Projection JRZ-08b represents JRZ-08 normalized with an additional 4 wt $\% \mathrm{Na}_{2} \mathrm{O}$. Projection JRZ-10 represents the SB6 blend with less washing in Tank 51 (wash through Decant I) and prior to the decant in Tank 40. Projection JRZ-11 represents the SB6 blend after the decant in Tank 40, again with less washing in Tank 51. 
Table 3-34. SB6 Composition Projections with Varying $\mathrm{Na}_{2} \mathrm{O}$ Concentrations.

\begin{tabular}{|c|c|c|c|}
\hline Oxide (wt \%) & JRZ-08b & JRZ-10 & JRZ-11 \\
\hline $\mathrm{Al}_{2} \mathrm{O}_{3}$ & 27.50 & 26.92 & 28.35 \\
\hline $\mathrm{BaO}$ & 0.14 & 0.14 & 0.15 \\
\hline $\mathrm{CaO}$ & 1.92 & 1.85 & 1.98 \\
\hline $\mathrm{CdO}$ & 0.11 & 0.11 & 0.11 \\
\hline $\mathrm{Ce}_{2} \mathrm{O}_{3}$ & 0.24 & 0.23 & 0.25 \\
\hline $\mathrm{Cr}_{2} \mathrm{O}_{3}$ & 0.15 & 0.15 & 0.16 \\
\hline $\mathrm{CuO}$ & 0.12 & 0.11 & 0.12 \\
\hline $\mathrm{Fe}_{2} \mathrm{O}_{3}$ & 26.13 & 25.07 & 26.87 \\
\hline $\mathrm{Gd}_{2} \mathrm{O}_{3}$ & 0.05 & 0.05 & 0.05 \\
\hline $\mathrm{K}_{2} \mathrm{O}$ & 0.06 & 0.08 & 0.07 \\
\hline $\mathrm{La}_{2} \mathrm{O}_{3}$ & 0.10 & 0.10 & 0.11 \\
\hline $\mathrm{MgO}$ & 0.72 & 0.69 & 0.74 \\
\hline $\mathrm{MnO}$ & 7.10 & 6.81 & 7.30 \\
\hline $\mathrm{Na}_{2} \mathrm{O}$ & 23.29 & 25.42 & 20.93 \\
\hline $\mathrm{NiO}$ & 3.28 & 3.15 & 3.37 \\
\hline $\mathrm{PbO}$ & 0.03 & 0.03 & 0.03 \\
\hline $\mathrm{SO}_{4}{ }^{2-}$ & 0.98 & 1.32 & 1.11 \\
\hline $\mathrm{SiO}_{2}$ & 2.45 & 2.37 & 2.52 \\
\hline $\mathrm{SrO}$ & 0.07 & 0.07 & 0.07 \\
\hline $\mathrm{TiO}_{2}$ & 0.02 & 0.02 & 0.02 \\
\hline $\mathrm{U}_{3} \mathrm{O}_{8}$ & 5.13 & 4.93 & 5.29 \\
\hline $\mathrm{ZnO}$ & 0.06 & 0.06 & 0.06 \\
\hline $\mathrm{ZrO}_{2}$ & 0.33 & 0.32 & 0.34 \\
\hline $\begin{array}{c}\text { Supernate } \mathrm{Na}^{+} \\
\text {(M) }\end{array}$ & $\mathrm{n} / \mathrm{a}$ & 1.047 & 1.040 \\
\hline $\begin{array}{c}\text { Insoluble } \\
\text { solids (wt \%) }\end{array}$ & $\mathrm{n} / \mathrm{a}$ & 9.45 & 11.96 \\
\hline
\end{tabular}

A Nominal Stage MAR assessment was completed using these three SB6 composition projections with and without the addition of ARP, and the array of 1,144 frit compositions described in Table 3-19. The results were reviewed to identify candidate frit compositions that provided projected operating windows of at least $30-40 \%$ WL, with 257 frits meeting this criterion for JRZ-08b, 279 frits for JRZ-10 and 157 frits for JRZ-11 (all with and without ARP). A much smaller number of frits were down-selected for each of the sludge composition projections for a Variation Stage assessment, using the sludge composition intervals given in Table 3-35. The compositions of these frits and the Variation Stage results are summarized in Table 3-36. 
Table 3-35. Sludge Composition Intervals for JRZ-08b, JRZ-10 and JRZ-11.

\begin{tabular}{|c|c|c|c|c|c|c||}
\hline \multirow{2}{*}{$\begin{array}{c}\text { Oxide } \\
\text { (wt \%) }\end{array}$} & \multicolumn{2}{|c|}{ JRZ-08b } & \multicolumn{2}{c|}{ JRZ-10 } & \multicolumn{2}{c|}{ JRZ-11 } \\
\cline { 2 - 7 } & Minimum & Maximum & Minimum & Maximum & Minimum & Maximum \\
\hline $\mathrm{Al}_{2} \mathrm{O}_{3}$ & 24.47 & 29.57 & 23.96 & 28.94 & 25.21 & 30.48 \\
\hline $\mathrm{CaO}$ & 1.38 & 2.42 & 1.30 & 2.35 & 1.43 & 2.48 \\
\hline $\mathrm{Fe}_{2} \mathrm{O}_{3}$ & 23.53 & 28.09 & 22.61 & 26.96 & 24.18 & 28.88 \\
\hline $\mathrm{MgO}$ & 0.19 & 1.22 & 0.17 & 1.19 & 0.21 & 1.24 \\
\hline $\mathrm{MnO}$ & 6.46 & 7.64 & 6.20 & 7.32 & 6.63 & 7.85 \\
\hline $\mathrm{Na}_{2} \mathrm{O}$ & 21.54 & 25.93 & 23.51 & 28.10 & 19.36 & 23.52 \\
\hline $\mathrm{NiO}$ & 2.69 & 3.78 & 2.56 & 3.65 & 2.77 & 3.87 \\
\hline $\mathrm{SO}_{4}{ }_{4}^{2-}$ & 0.73 & 1.32 & 1.07 & 1.64 & 0.86 & 1.44 \\
\hline $\mathrm{SiO}_{2}$ & 1.86 & 2.95 & 1.78 & 2.87 & 1.93 & 3.02 \\
\hline $\mathrm{TiO}_{2}$ & 0.00 & 1.81 & 0.00 & 1.81 & 0.00 & 1.81 \\
\hline $\mathrm{U}_{3} \mathrm{O}_{8}$ & 4.54 & 5.63 & 4.35 & 5.43 & 4.69 & 5.79 \\
\hline $\mathrm{Others}_{2}$ & 0.73 & 1.76 & 0.71 & 1.74 & 0.77 & 1.80 \\
\hline
\end{tabular}

Table 3-36. Variation Stage Assessment Results for Sludge Compositions with Varying $\mathrm{Na}_{2} \mathrm{O}$ Concentrations.

\begin{tabular}{|c|c|c|c|c|c|}
\hline $\begin{array}{c}\begin{array}{c}\text { Frit Oxides } \\
(w \mathrm{t} \%)\end{array} \\
\end{array}$ & JRZ-08b & $\begin{array}{c}\begin{array}{c}\text { Frit Oxides } \\
(w t \%)\end{array} \\
\end{array}$ & JRZ-10 & $\begin{array}{c}\text { Frit Oxides } \\
(\text { wt \%) }\end{array}$ & JRZ-11 \\
\hline $\begin{array}{c}\text { B-8;Li-8; } \\
\text { Na-8;Si-76 } \\
\text { (Frit 418) }\end{array}$ & $\begin{array}{c}25-40 \\
\mathrm{~T}_{\mathrm{L}}, \mathrm{Neph} \\
41\end{array}$ & $\begin{array}{c}\text { B-8;Li-8; } \\
\text { Na-8;Si-76 } \\
\text { (Frit 418) }\end{array}$ & $\begin{array}{c}- \\
25-39 \\
\text { Neph } \\
40\end{array}$ & $\begin{array}{c}\text { B-8;Li-8; } \\
\text { Na-8;Si-76 } \\
\text { (Frit 418) }\end{array}$ & $\begin{array}{c}\text { highv } \\
27-37 \\
\mathrm{~T}_{\mathrm{L}} \\
42\end{array}$ \\
\hline $\begin{array}{l}\text { B-10;Li-10; } \\
\text { Na-5;Si-75 }\end{array}$ & $\begin{array}{c}- \\
25-38 \\
\mathrm{~T}_{\mathrm{L}} \\
42 \\
\end{array}$ & $\begin{array}{l}\text { B-11;Li-10; } \\
\text { Na-2;Si-77 }\end{array}$ & $\begin{array}{c}- \\
25-38 \\
\mathrm{~T}_{\mathrm{L}} \\
44 \\
\end{array}$ & $\begin{array}{l}\text { B-8;Li-11; } \\
\text { Na-6;Si-75 }\end{array}$ & $\begin{array}{c}- \\
25-38 \\
\mathrm{~T}_{\mathrm{L}} \\
42 \\
\end{array}$ \\
\hline $\begin{array}{l}\text { B-10;Li-11: } \\
\text { Na-4;Si-75 }\end{array}$ & $\begin{array}{c}- \\
25-38 \\
\mathrm{~T}_{\mathrm{L}} \\
38 \\
\end{array}$ & $\begin{array}{l}\text { B-12;Li-11; } \\
\text { Na-2;Si-75 }\end{array}$ & $\begin{array}{c}- \\
25-39 \\
\mathrm{~T}_{\mathrm{L}}, \text { lowv } \\
43\end{array}$ & $\begin{array}{l}\text { B-8;Li-11; } \\
\text { Na-7;Si-74 }\end{array}$ & $\begin{array}{c}- \\
25-37 \\
\text { lowv } \\
41 \\
\end{array}$ \\
\hline $\begin{array}{l}\text { B-11;Li-11; } \\
\text { Na-3;Si-75 }\end{array}$ & $\begin{array}{c}- \\
25-37 \\
\mathrm{~T}_{\mathrm{L}} \\
44 \\
\end{array}$ & $\begin{array}{l}\text { B-8;Li-12; } \\
\text { Na-2;Si-78 }\end{array}$ & $\begin{array}{c}- \\
25-40 \\
\mathrm{~T}_{\mathrm{L}} \\
44 \\
\end{array}$ & $\begin{array}{l}\text { B-8;Li-9; } \\
\text { Na-7;Si-76 }\end{array}$ & $\begin{array}{c}- \\
25-37 \\
\mathrm{~T}_{\mathrm{L}} \\
42 \\
\end{array}$ \\
\hline $\begin{array}{l}\text { B-8;Li-11; } \\
\text { Na-4;Si-77 }\end{array}$ & $\begin{array}{c}- \\
25-39 \\
\mathrm{~T}_{\mathrm{L}} \\
44 \\
\end{array}$ & $\begin{array}{l}\text { B-9;Li-9; } \\
\text { Na-3;Si-79 }\end{array}$ & $\begin{array}{c}\text { highv } \\
35-38 \\
\mathrm{~T}_{\mathrm{L}} \\
44 \\
\end{array}$ & $\begin{array}{l}\text { B-9;Li-10; } \\
\text { Na-7;Si-74 }\end{array}$ & $\begin{array}{c}- \\
25-38 \\
\mathrm{~T}_{\mathrm{L}} \\
41 \\
\end{array}$ \\
\hline $\begin{array}{l}\text { B-8;Li-12; } \\
\text { Na-4;Si-76 }\end{array}$ & $\begin{array}{c}- \\
25-38 \\
\text { lowv } \\
43 \\
\end{array}$ & & & & \\
\hline
\end{tabular}

In summary, the performance of Frit 418 is significantly improved for composition JRZ-08b as compared to JRZ-08 (refer to Table 3-25). In other words, a higher $\mathrm{Na}_{2} \mathrm{O}$ concentration in the sludge after the Tank 40 decant helps improve the projected operating window for Frit 418 . The other frits assessed with JRZ-08b did not provide larger projected operating windows than 
Frit 418, although some were able to push nepheline crystallization predictions to higher waste loadings. For composition JRZ-10, there was no improvement in performance with Frit 418 as compared with composition JRZ-07 (refer to Table 3-25), indicating that less washing in Tank 51 has little impact on the projected operating windows prior to the Tank 40 decant. The other frits assessed with JRZ-10 again were able to push nepheline crystallization predictions to higher waste loadings, but provide no practical improvement in projected operating windows. The results of the frits assessed with JRZ-11 show basically no improvement in performance as compared to previous projections of the post Tank 40 decant SB6 composition.

One additional SB6 composition projection, JRZ-11b, was developed to determine whether additional $\mathrm{Na}_{2} \mathrm{O}$ in the sludge after the decant in Tank 40 would be beneficial for frit performance. This projection is based on JRZ-11, normalized with an additional $4 \mathrm{wt} \% \mathrm{Na}_{2} \mathrm{O}$, as shown in Table 3-37. A Nominal Stage MAR assessment was completed using the frit array described in Table 3-19. A brief review of the Nominal Stage results showed that 280 frit compositions were available that provided a projected operating window of at least $30-40 \% \mathrm{WL}$. A small number of frits, shown in Table 3-38, were selected for a variation stage assessment to gauge any improvement in projected operating window. The sludge composition intervals developed for the Variation Stage assessment are given in Table 3-39.

Table 3-37. Composition of SB6 Projection JRZ-11b.

\begin{tabular}{|c|c|}
\hline Oxide (wt \%) & JRZ-11b \\
\hline $\mathrm{Al}_{2} \mathrm{O}_{3}$ & 26.92 \\
\hline $\mathrm{BaO}$ & 0.14 \\
\hline $\mathrm{CaO}$ & 1.88 \\
\hline $\mathrm{CdO}$ & 0.11 \\
\hline $\mathrm{Ce}_{2} \mathrm{O}_{3}$ & 0.24 \\
\hline $\mathrm{Cr}_{2} \mathrm{O}_{3}$ & 0.15 \\
\hline $\mathrm{CuO}$ & 0.12 \\
\hline $\mathrm{Fe}_{2} \mathrm{O}_{3}$ & 25.51 \\
\hline $\mathrm{Gd}_{2} \mathrm{O}_{3}$ & 0.05 \\
\hline $\mathrm{K}_{2} \mathrm{O}$ & 0.07 \\
\hline $\mathrm{La}_{2} \mathrm{O}_{3}$ & 0.10 \\
\hline $\mathrm{MgO}$ & 0.71 \\
\hline $\mathrm{MnO}$ & 6.93 \\
\hline $\mathrm{Na}_{2} \mathrm{O}$ & 24.93 \\
\hline $\mathrm{NiO}$ & 3.20 \\
\hline $\mathrm{PbO}$ & 0.03 \\
\hline $\mathrm{SO}_{4}{ }^{2-}$ & 1.05 \\
\hline $\mathrm{SiO} O_{2}$ & 2.40 \\
\hline $\mathrm{SrO}$ & 0.07 \\
\hline $\mathrm{TiO} \mathrm{O}_{2}$ & 0.02 \\
\hline $\mathrm{U}_{3} \mathrm{O}_{8}$ & 5.02 \\
\hline $\mathrm{ZnO}$ & 0.06 \\
\hline $\mathrm{ZrO}$ & 0.32 \\
\hline
\end{tabular}


SRNL-STI-2010-00137

Revision 0

Table 3-38. Frit Compositions Selected for a Variation Stage Assessment with JRZ-11b.

\begin{tabular}{||c|c|c|c|}
\hline $\mathbf{B}_{\mathbf{2}} \mathbf{O}_{\mathbf{3}}$ & $\mathbf{L i}_{\mathbf{2}} \mathbf{O}$ & $\mathbf{N a}_{\mathbf{2}} \mathbf{O}$ & $\mathbf{S i O}_{\mathbf{2}}$ \\
\hline 10 & 11 & 3 & 76 \\
\hline 10 & 9 & 5 & 76 \\
\hline 14 & 10 & 2 & 74 \\
\hline 8 & 10 & 4 & 78 \\
\hline 8 & 12 & 2 & 78 \\
\hline 8 & 8 & 8 & 76 \\
\hline
\end{tabular}

Table 3-39. Sludge Composition Intervals for JRZ-11b.

\begin{tabular}{||c|c|c||}
\hline \hline \multirow{2}{*}{$\begin{array}{c}\text { Oxide } \\
\text { (wt \%) }\end{array}$} & \multicolumn{2}{|c||}{ JRZ-11b } \\
\cline { 2 - 3 } & Minimum & Maximum \\
\hline $\mathrm{Al}_{2} \mathrm{O}_{3}$ & 23.95 & 28.94 \\
\hline $\mathrm{CaO}$ & 1.34 & 2.38 \\
\hline $\mathrm{Fe}_{2} \mathrm{O}_{3}$ & 22.99 & 27.42 \\
\hline $\mathrm{MgO}$ & 0.18 & 1.21 \\
\hline $\mathrm{MnO}$ & 6.30 & 7.45 \\
\hline $\mathrm{Na}_{2} \mathrm{O}$ & 23.06 & 27.61 \\
\hline $\mathrm{NiO}$ & 2.61 & 3.70 \\
\hline $\mathrm{SO}_{4}{ }^{2-}$ & 0.80 & 1.39 \\
\hline $\mathrm{SiO}_{2}$ & 1.81 & 2.90 \\
\hline $\mathrm{TiO}_{2}$ & 0.00 & 1.81 \\
\hline $\mathrm{U}_{3} \mathrm{O}_{8}$ & 4.43 & 5.52 \\
\hline $\mathrm{Others}_{2}$ & 0.71 & 1.73 \\
\hline \multicolumn{2}{|l}{} \\
\hline
\end{tabular}

The results of the Variation Stage assessment for JRZ-11b are shown in Table 3-40. In summary, the performance of Frit 418 is improved with JRZ-11b as compared with JRZ-11 (refer to Table 3-36). Increasing the $\mathrm{Na}_{2} \mathrm{O}$ concentration in the sludge after the Tank 40 decant would be beneficial in improving the projected operating windows. The results for some of the other frits in Table 3-40 show that projected operating windows equivalent to that of Frit 418 are available for systems that are limited by liquidus temperature predictions rather than predictions of nepheline crystallization, which would be advantageous since liquidus temperature is not a waste form affecting constraint. Overall, the projected operating windows for the various SB6 projections with several different groups of frits remain small considering that the nominal, target waste loading will be $36 \%$. One additional frit composition was added to the melt rate study to provide performance data for a frit with a low $\mathrm{Na}_{2} \mathrm{O}$ concentration. This frit is labeled IS7 in Table 3-40. 
SRNL-STI-2010-00137

Revision 0

Table 3-40. Variation Stage Assessment Results for JRZ-11b.

\begin{tabular}{|c|c|c|c|}
\hline $\begin{array}{c}\text { Frit Oxides } \\
(\text { wt \%) }\end{array}$ & JRZ-11b & $\begin{array}{c}\text { Frit Oxides } \\
(\text { wt } \%)\end{array}$ & JRZ-11b \\
\hline $\begin{array}{c}\text { B-8;Li-8; } \\
\text { Na-8;Si-76 } \\
\text { (Frit 418) }\end{array}$ & $\begin{array}{c}- \\
25-39 \\
\text { Neph } \\
40 \\
\end{array}$ & $\begin{array}{l}\text { B-14;Li-10; } \\
\text { Na-2;Si-74 }\end{array}$ & $\begin{array}{c}- \\
25-37 \\
\mathrm{~T}_{\mathrm{L}} \\
43 \\
\end{array}$ \\
\hline $\begin{array}{l}\text { B-10;Li-11; } \\
\text { Na-3;Si-76 }\end{array}$ & $\begin{array}{c}- \\
25-39 \\
\mathrm{~T}_{\mathrm{L}} \\
43 \\
\end{array}$ & $\begin{array}{l}\text { B-8;Li-10; } \\
\text { Na-4;Si-78 }\end{array}$ & $\begin{array}{c}- \\
25-39 \\
\mathrm{~T}_{\mathrm{L}} \\
44 \\
\end{array}$ \\
\hline $\begin{array}{l}\text { B-10;Li-9; } \\
\text { Na-5;Si-76 }\end{array}$ & $\begin{array}{c}- \\
25-39 \\
\mathrm{~T}_{\mathrm{L}} \\
42\end{array}$ & $\begin{array}{l}\text { B-8; } \mathrm{Li}-12 ; \\
\text { Na-2;Si-78 } \\
\text { (IS7) }\end{array}$ & $\begin{array}{c}- \\
25-39 \\
\mathrm{~T}_{\mathrm{L}} \\
45\end{array}$ \\
\hline
\end{tabular}

\subsection{Late January 2010 SB6 Composition Projections}

Two new SB6 composition projections were received from SRR on January 25, 2010. The changes again accounted for the reduction in the number of SB6 washes as recommended by SRNL to increase the sludge $\mathrm{Na}_{2} \mathrm{O}$ concentration, as well as analyses of the SB6 qualification sample in the SRNL shielded cells. The projections were labeled JanSB6-01, representing the SB6 blend prior to the decant in Tank 40, and JanSB6-02, representing the SB6 blend after the Tank 40 decant. The projections were converted to oxides and normalized to $100 \mathrm{wt} \%$, as shown in Table 3-41. The impact of ARP additions was also calculated for each of the projections. 
SRNL-STI-2010-00137

Revision 0

Table 3-41. SB6 Composition Projections JanSB6-01 and JanSB6-02.

\begin{tabular}{|c|c|c|}
\hline Oxide (wt \%) & JanSB6-01 & JanSB6-02 \\
\hline $\mathrm{Al}_{2} \mathrm{O}_{3}$ & 24.93 & 26.44 \\
\hline $\mathrm{BaO}$ & 0.15 & 0.16 \\
\hline $\mathrm{CaO}$ & 1.51 & 1.66 \\
\hline $\mathrm{Ce}_{2} \mathrm{O}_{3}$ & 0.21 & 0.23 \\
\hline $\mathrm{Cr}_{2} \mathrm{O}_{3}$ & 0.35 & 0.39 \\
\hline $\mathrm{CuO}$ & 0.11 & 0.13 \\
\hline $\mathrm{Fe}_{2} \mathrm{O}_{3}$ & 24.92 & 27.31 \\
\hline $\mathrm{K}_{2} \mathrm{O}$ & 0.02 & 0.02 \\
\hline $\mathrm{La}_{2} \mathrm{O}_{3}$ & 0.11 & 0.12 \\
\hline $\mathrm{MgO}$ & 0.81 & 0.90 \\
\hline $\mathrm{MnO}$ & 7.14 & 7.82 \\
\hline $\mathrm{Na}_{2} \mathrm{O}$ & 27.33 & 21.56 \\
\hline $\mathrm{NiO}$ & 3.07 & 3.36 \\
\hline $\mathrm{PbO}$ & 0.03 & 0.03 \\
\hline $\mathrm{SO}_{4}{ }^{2-}$ & 1.41 & 1.20 \\
\hline $\mathrm{SiO}_{2}$ & 2.29 & 2.52 \\
\hline $\mathrm{ThO}_{2}$ & 0.02 & 0.03 \\
\hline $\mathrm{TiO}_{2}$ & 0.03 & 0.05 \\
\hline $\mathrm{U}_{3} \mathrm{O}_{8}$ & 5.11 & 5.59 \\
\hline $\mathrm{ZnO}$ & 0.09 & 0.10 \\
\hline $\mathrm{ZrO}_{2}$ & 0.35 & 0.38 \\
\hline $\begin{array}{c}\text { Supernate } \mathrm{Na}^{+} \\
(\mathrm{M})\end{array}$ & 0.963 & 0.919 \\
\hline $\begin{array}{c}\text { Insoluble solids } \\
\text { (wt \%) }\end{array}$ & 8.39 & 8.80 \\
\hline
\end{tabular}

The sludges described in Table 3-41 were combined with the array of 1,144 frit compositions in Table 3-19 for a Nominal Stage MAR assessment. Briefly, 250 frits are available for JanSB6-01 that provide projected operating windows of at least $30-40 \% \mathrm{WL}$ with and without ARP, some of which provide maximum waste loadings of $45 \%$. Only 44 frit compositions are available that meet this criterion for JanSB6-02, and the maximum waste loading for any of these frits at the Nominal Stage is $41 \%$.

Next, a larger frit array was developed for use in evaluating the JanSB6-02 projection to determine whether any frits could be identified that would provide wider projected operating windows. The frit array was expanded to include $\mathrm{CaO}, \mathrm{Fe}_{2} \mathrm{O}_{3}, \mathrm{MgO}$ and $\mathrm{ZrO}_{2}$, as shown in Table 3-42. These frits were combined with the JanSB6-02 projection for a second Nominal Stage MAR assessment. The impact of ARP was not included for this assessment due to the large number of frit combinations $(353,430)$. The results of the Nominal Stage assessment showed that 234 of the frits provided projected operating windows of at least 30-40\% WL with the JanSB6-02 projection. However, none of the frits provided maximum waste loadings of more that $41 \%$. The projected SB6 composition after the decant in Tank 40 continues to be a challenge for a targeted waste loading of $36 \%$. 
Table 3-42. Expanded Frit Array for JanSB6-02.

\begin{tabular}{||c|c|c|c|c|c|c|c|c||}
\hline & $\mathbf{B}_{\mathbf{2}} \mathbf{O}_{\mathbf{3}}$ & $\mathbf{C a O}$ & $\mathbf{F e}_{\mathbf{2}} \mathbf{O}_{\mathbf{3}}$ & $\mathbf{L i}_{\mathbf{2}} \mathbf{O}$ & $\mathbf{M g O}$ & $\mathbf{N a}_{\mathbf{2}} \mathbf{O}$ & $\mathbf{S i O}_{\mathbf{2}}$ & $\mathbf{Z r O}_{\mathbf{2}}$ \\
\hline Minimum (wt \%) & 8.0 & 0.0 & 0.0 & 6.0 & 0.0 & 4.0 & 37.0 & 0.0 \\
\hline Maximum (wt \%) & 12.0 & 5.0 & 10.0 & 12.0 & 2.0 & 20.0 & 82.0 & 2.0 \\
\hline \hline
\end{tabular}

\subsection{February 2010 SB6 Composition Projections}

SRNL developed a revised series of SB6 composition projections in early February 2010 based on the measured composition of the Tank 51 qualification sample. ${ }^{6}$ The projections included several potential blending scenarios to account for uncertainty in the timing of the SB6 transfer from Tank 51 to Tank 40. Identifiers and descriptions for these projections are given in Table 3-43, and their compositions as normalized oxides are shown in Table 3-44. It is important to note that these projections do not account for any decant occurring in Tank 40 during processing of SB6.

Table 3-43. Descriptions of the February SB6 Composition Projections.

\begin{tabular}{|c|l||}
\hline $\begin{array}{c}\text { SB6 Composition } \\
\text { Projection }\end{array}$ & \multicolumn{1}{c|}{ Description } \\
\hline FebSB6-62 & SB6 Blend with a 62 inch SB5 heel remaining in Tank 40 \\
\hline FebSB6-51 & SB6 Blend with a 51 inch SB5 heel remaining in Tank 40 \\
\hline FebSB6-40 & SB6 Blend with a 40 inch SB5 heel remaining in Tank 40 \\
\hline FebSB6-40a & $\begin{array}{l}\text { SB5 processed until Tank 40 reaches 40 inches, water added to allow } \\
\text { one additional SB5 feed to DWPF, Tank 40 again reduced to 40 inches }\end{array}$ \\
\hline FebSB6-40b & $\begin{array}{l}\text { Similar to FebSB6-40a, with one added iteration of water additions and } \\
\text { feeding to DWPF. }\end{array}$ \\
\hline FebSB6-40c & $\begin{array}{l}\text { Similar to FebSB6-40a, with two added iterations of water additions } \\
\text { and feeding to DWPF. }\end{array}$ \\
\hline FebSB6-40d & $\begin{array}{l}\text { Similar to FebSB6-40a, with three added iterations of water additions } \\
\text { and feeding to DWPF. }\end{array}$ \\
\hline
\end{tabular}


Table 3-44. Compositions of the February SB6 Projections.

\begin{tabular}{|c|c|c|c|c|c|c|c|}
\hline $\begin{array}{c}\text { Oxide } \\
(\text { wt \%) }\end{array}$ & $\begin{array}{c}\text { FebSB6- } \\
62\end{array}$ & $\begin{array}{c}\text { FebSB6- } \\
51\end{array}$ & $\begin{array}{c}\text { FebSB6- } \\
40\end{array}$ & $\begin{array}{c}\text { FebSB6- } \\
40 a\end{array}$ & $\begin{array}{c}\text { FebSB6- } \\
40 b\end{array}$ & $\begin{array}{c}\text { FebSB6- } \\
40 c\end{array}$ & $\begin{array}{c}\text { FebSB6- } \\
\text { 40d }\end{array}$ \\
\hline $\mathrm{Al}_{2} \mathrm{O}_{3}$ & 26.39 & 26.61 & 26.89 & 26.98 & 27.06 & 27.14 & 27.21 \\
\hline $\mathrm{BaO}$ & 0.13 & 0.14 & 0.14 & 0.14 & 0.14 & 0.14 & 0.14 \\
\hline $\mathrm{CaO}$ & 1.53 & 1.46 & 1.39 & 1.36 & 1.34 & 1.31 & 1.29 \\
\hline $\mathrm{CdO}$ & 0.04 & 0.04 & 0.03 & 0.03 & 0.03 & 0.03 & 0.03 \\
\hline $\mathrm{Ce}_{2} \mathrm{O}_{3}$ & 0.21 & 0.21 & 0.21 & 0.21 & 0.21 & 0.21 & 0.21 \\
\hline $\mathrm{Cr}_{2} \mathrm{O}_{3}$ & 0.13 & 0.13 & 0.12 & 0.12 & 0.12 & 0.11 & 0.11 \\
\hline $\mathrm{CuO}$ & 0.11 & 0.11 & 0.11 & 0.11 & 0.11 & 0.11 & 0.11 \\
\hline $\mathrm{Fe}_{2} \mathrm{O}_{3}$ & 24.95 & 24.69 & 24.43 & 24.31 & 24.21 & 24.12 & 24.03 \\
\hline $\mathrm{Gd}_{2} \mathrm{O}_{3}$ & 0.11 & 0.11 & 0.11 & 0.11 & 0.11 & 0.12 & 0.12 \\
\hline $\mathrm{K}_{2} \mathrm{O}$ & 0.08 & 0.08 & 0.09 & 0.09 & 0.09 & 0.09 & 0.09 \\
\hline $\mathrm{La}_{2} \mathrm{O}_{3}$ & 0.11 & 0.11 & 0.11 & 0.11 & 0.11 & 0.11 & 0.11 \\
\hline $\mathrm{MgO}$ & 0.88 & 0.83 & 0.77 & 0.75 & 0.73 & 0.72 & 0.70 \\
\hline $\mathrm{MnO}$ & 6.87 & 6.92 & 6.98 & 7.00 & 7.02 & 7.03 & 7.05 \\
\hline $\mathrm{MoO}_{3}$ & 0.01 & 0.01 & 0.01 & 0.01 & 0.01 & 0.01 & 0.01 \\
\hline $\mathrm{Na}_{2} \mathrm{O}$ & 25.86 & 26.14 & 26.39 & 26.52 & 26.63 & 26.73 & 26.83 \\
\hline $\mathrm{NiO}$ & 3.01 & 3.00 & 2.99 & 2.98 & 2.98 & 2.97 & 2.97 \\
\hline $\mathrm{P}_{2} \mathrm{O}_{5}$ & 0.46 & 0.46 & 0.46 & 0.46 & 0.46 & 0.45 & 0.45 \\
\hline $\mathrm{PbO}$ & 0.03 & 0.03 & 0.03 & 0.03 & 0.03 & 0.03 & 0.03 \\
\hline $\mathrm{SO}_{4}{ }^{2-}$ & 1.31 & 1.34 & 1.37 & 1.38 & 1.39 & 1.40 & 1.41 \\
\hline $\mathrm{SiO}_{2}$ & 2.24 & 2.22 & 2.20 & 2.19 & 2.18 & 2.17 & 2.16 \\
\hline $\mathrm{SrO}$ & 0.07 & 0.07 & 0.07 & 0.07 & 0.07 & 0.07 & 0.07 \\
\hline $\mathrm{TiO}_{2}$ & 0.04 & 0.04 & 0.04 & 0.04 & 0.04 & 0.04 & 0.04 \\
\hline $\mathrm{U}_{3} \mathrm{O}_{8}$ & 5.15 & 4.97 & 4.78 & 4.70 & 4.63 & 4.57 & 4.51 \\
\hline $\mathrm{ZnO}$ & 0.07 & 0.07 & 0.07 & 0.07 & 0.07 & 0.07 & 0.07 \\
\hline $\mathrm{ZrO}_{2}$ & 0.22 & 0.23 & 0.24 & 0.24 & 0.24 & 0.25 & 0.25 \\
\hline $\begin{array}{l}\text { Supernate } \\
\mathrm{Na}^{+}(\mathrm{M})\end{array}$ & 0.879 & 0.879 & 0.879 & 0.879 & 0.879 & 0.879 & 0.879 \\
\hline $\begin{array}{c}\text { Insoluble } \\
\text { solids (wt \%) }\end{array}$ & 10.26 & 10.26 & 10.26 & 9.46 & 8.70 & 8.01 & 7.36 \\
\hline
\end{tabular}

The impact of ARP additions was calculated for each of these projections, resulting in 14 compositions for a Nominal Stage MAR assessment with the array of 1,144 frits described in Table 3-19. The results of the Nominal Stage assessment were reviewed to identify frits that provide projected operating windows of at least 30-40\% WL for all 14 scenarios, with 252 candidate frits meeting this criterion. The Nominal Stage results for Frit 418 are summarized in Table 3-45, and show a slight improvement in projected operating window as compared to earlier SB6 composition projections, regardless of when the transfer to Tank 40 occurs.

Table 3-45. Summary of Nominal Stage Assessment Results for Frit 418 with the FebSB6-series composition projections.

\begin{tabular}{||c|c||}
\hline & $\begin{array}{c}\text { Summary for all } \\
\text { FebSB6-series sludge } \\
\text { composition projections }\end{array}$ \\
\hline & - \\
Frit 418 & $25-41$ \\
& Neph \\
& 42 \\
\hline
\end{tabular}


A group of additional frits was down-selected from the Nominal Stage results to determine whether projected operating windows better than those provided by Frit 418 are available when variation is applied to the sludge composition. These frits are listed in Table 3-46.

Table 3-46. Additional Frit Compositions for the FebSB6-series Projections.

\begin{tabular}{||c|c|c|c||}
\hline $\mathbf{B}_{\mathbf{2}} \mathbf{O}_{\mathbf{3}}$ & $\mathbf{L i}_{\mathbf{2}} \mathbf{O}$ & $\mathbf{N a}_{\mathbf{2}} \mathbf{O}$ & $\mathbf{S i O}_{\mathbf{2}}$ \\
\hline 8 & 8 & 8 & 76 \\
\hline 10 & 11 & 2 & 77 \\
\hline 11 & 10 & 2 & 77 \\
\hline 8 & 10 & 2 & 80 \\
\hline 8 & 12 & 2 & 78 \\
\hline 9 & 11 & 2 & 78 \\
\hline
\end{tabular}

Sludge composition intervals were developed for a Variation Stage assessment encompassing all of the FebSB6-series composition projections, both with and without ARP, as shown in Table 3-47. The EVs of this region were then identified and combined with the frit compositions in Table 3-46 over waste loadings of 25-50\%. Each glass composition was then evaluated against the PCCS MAR, and the results are given in Table 3-48.

Table 3-47. Sludge Composition Intervals for the FebSB6-series of Composition Projections.

\begin{tabular}{|c|c|c||}
\hline \multirow{2}{*}{$\begin{array}{c}\text { Oxide } \\
\text { wt \%) }\end{array}$} & \multicolumn{2}{|c|}{ FebSB6-series } \\
\cline { 2 - 3 } & Minimum & Maximum \\
\hline $\mathrm{Al}_{2} \mathrm{O}_{3}$ & 23.49 & 29.25 \\
\hline $\mathrm{CaO}$ & 0.78 & 2.03 \\
\hline $\mathrm{Fe}_{2} \mathrm{O}_{3}$ & 21.69 & 26.82 \\
\hline $\mathrm{MgO}$ & 0.17 & 1.38 \\
\hline $\mathrm{MnO}$ & 6.25 & 7.58 \\
\hline $\mathrm{Na}_{2} \mathrm{O}$ & 23.92 & 29.55 \\
\hline $\mathrm{NiO}^{2-}$ & 2.39 & 3.51 \\
\hline $\mathrm{SO}_{4}{ }^{2-}$ & 1.06 & 1.73 \\
\hline $\mathrm{SiO}_{2}$ & 1.59 & 2.74 \\
\hline $\mathrm{TiO}_{2}$ & 0.00 & 1.84 \\
\hline $\mathrm{U}_{3} \mathrm{O}_{8}$ & 3.95 & 5.65 \\
\hline $\mathrm{Others}_{2}$ & 0.56 & 1.65 \\
\hline
\end{tabular}


SRNL-STI-2010-00137

Revision 0

Table 3-48. Variation Stage Results for the FebSB6-series Projections with Select Frits.

\begin{tabular}{|c|c|c|c|}
\hline $\begin{array}{l}\text { Frit Oxides } \\
\quad(w t \%)\end{array}$ & $\begin{array}{c}\text { B-8;Li-8; } \\
\text { Na-8;Si-76 } \\
\text { (Frit 418) }\end{array}$ & $\begin{array}{l}\text { B-10;Li-11; } \\
\text { Na-2;Si-77 }\end{array}$ & $\begin{array}{l}\text { B-11;Li-10; } \\
\text { Na-2;Si-77 }\end{array}$ \\
\hline $\begin{array}{l}\text { Variation Stage } \\
\text { MAR Outcome }\end{array}$ & $\begin{array}{c}- \\
25-38 \\
\text { Neph } \\
39\end{array}$ & $\begin{array}{c}- \\
25-40 \\
\mathrm{~T}_{\mathrm{L}}, \text { lowv } \\
43\end{array}$ & $\begin{array}{c}- \\
25-39 \\
T_{L} \\
43\end{array}$ \\
\hline $\begin{array}{c}\text { Frit Oxides } \\
(\text { wt } \%)\end{array}$ & $\begin{array}{l}\text { B-8;Li-10; } \\
\text { Na-2;Si-80 }\end{array}$ & $\begin{array}{l}\text { B-8;Li-12; } \\
\text { Na-2;Si-78 }\end{array}$ & $\begin{array}{l}\text { B-9;Li-11; } \\
\text { Na-2;Si-78 }\end{array}$ \\
\hline $\begin{array}{l}\text { Variation Stage } \\
\text { MAR Outcome }\end{array}$ & $\begin{array}{c}\text { highv } \\
34-39 \\
\mathrm{~T}_{\mathrm{L}} \\
44\end{array}$ & $\begin{array}{c}- \\
25-39 \\
\text { lowv } \\
44\end{array}$ & $\begin{array}{c}- \\
25-40 \\
\mathrm{~T}_{\mathrm{L}} \\
44\end{array}$ \\
\hline
\end{tabular}

The results in Table 3-48 show that frits other than Frit 418 are available that can provide access to slightly higher waste loadings where all of the EVs are predicted to produce acceptable glasses, and perhaps more importantly, push predictions of nepheline crystallization to higher waste loadings. Both of these factors would be beneficial in targeting 36\% waste loading for SB6. Again, it is important to keep in mind that these projections do not account for any decant occurring in Tank 40 during processing of SB6.

Note that the frits other than 418 selected above have relatively low $\mathrm{Na}_{2} \mathrm{O}$ concentrations, which could potentially reduce melt rate. ${ }^{7}$ To balance this, a second set of frits with moderately higher $\mathrm{Na}_{2} \mathrm{O}$ concentrations was down-selected from the Nominal Stage results, as shown in Table 3-49. These frits provide projected operating windows up to at least $43 \% \mathrm{WL}$ for all of the projections at the Nominal Stage.

Table 3-49. Frits with Moderately Higher $\mathrm{Na}_{2} \mathrm{O}$ Concentrations for the FebSB6-series Projections.

\begin{tabular}{|c|c|c|c||}
\hline $\mathbf{B}_{\mathbf{2}} \mathbf{O}_{\mathbf{3}}$ & $\mathbf{L i}_{\mathbf{2}} \mathbf{O}$ & $\mathbf{N a}_{\mathbf{2}} \mathbf{O}$ & $\mathbf{S i O}_{\mathbf{2}}$ \\
\hline 10 & 10 & 4 & 76 \\
\hline 10 & 8 & 5 & 77 \\
\hline 12 & 9 & 4 & 75 \\
\hline 14 & 7 & 4 & 75 \\
\hline 8 & 10 & 5 & 77 \\
\hline 8 & 8 & 6 & 78 \\
\hline
\end{tabular}

A Variation Stage assessment was performed with the frits in Table 3-49 with same set of EVs, and the results are given in Table 3-50. Similar projected operating windows over which all the EVs are predicted to produce acceptable glasses are available with these frits, however the increased $\mathrm{Na}_{2} \mathrm{O}$ concentration moves nepheline predictions to lower waste loadings. This may be acceptable (assuming a target waste loading of 36\% for SB6) if the increased $\mathrm{Na}_{2} \mathrm{O}$ concentration in these frits relative to the $2 \mathrm{wt} \% \mathrm{Na}_{2} \mathrm{O}$ frits can improve melt rate. 
Table 3-50. Variation Stage Results for the FebSB6-series Projections with Moderately Higher $\mathrm{Na}_{2} \mathrm{O}$ Concentration Frits.

\begin{tabular}{|c|c|c|c|}
\hline $\begin{array}{c}\text { Frit Oxides } \\
(w t \%)\end{array}$ & $\begin{array}{l}\text { B-10;Li-10; } \\
\text { Na-4;Si-76 }\end{array}$ & $\begin{array}{l}\text { B-10;Li-8; } \\
\text { Na-5;Si-77 }\end{array}$ & $\begin{array}{l}\text { B-12;Li-9; } \\
\text { Na-4;Si-75 }\end{array}$ \\
\hline $\begin{array}{l}\text { Variation Stage } \\
\text { MAR Outcome }\end{array}$ & $\begin{array}{c}- \\
25-39 \\
\text { lowv } \\
42 \\
\end{array}$ & $\begin{array}{c}\text { highv } \\
31-40 \\
\mathrm{~T}_{\mathrm{L}} \\
42 \\
\end{array}$ & $\begin{array}{c}- \\
25-40 \\
\mathrm{~T}_{\mathrm{L}}, \mathrm{Neph}, \text { lowv } \\
41 \\
\end{array}$ \\
\hline $\begin{array}{c}\text { Frit Oxides } \\
(w t \%)\end{array}$ & $\begin{array}{l}\text { B-14;Li-7; } \\
\text { Na-4;Si-75 }\end{array}$ & $\begin{array}{l}\text { B-8;Li-10; } \\
\text { Na-5;Si-77 }\end{array}$ & $\begin{array}{c}\text { B-8;Li-8; } \\
\text { Na-6;Si-78 }\end{array}$ \\
\hline $\begin{array}{l}\text { Variation Stage } \\
\text { MAR Outcome }\end{array}$ & $\begin{array}{c}\text { highv } \\
33-37 \\
\mathrm{~T}_{\mathrm{L}} \\
41 \\
\end{array}$ & $\begin{array}{c}- \\
25-40 \\
\text { lowv } \\
42 \\
\end{array}$ & $\begin{array}{c}\text { highv } \\
32-40 \\
\text { Neph } \\
41 \\
\end{array}$ \\
\hline
\end{tabular}

One additional approach was taken with these SB6 composition projections in an attempt to locate frit compositions that can provide further improvements in projected operating windows. The frit composition arrays used so far in this study have assumed that some $\mathrm{Na}_{2} \mathrm{O}$ concentration must be included in the frit. This may not be necessary due to the relatively high $\mathrm{Na}_{2} \mathrm{O}$ concentrations in the revised projections for SB6. Therefore, an additional Nominal Stage assessment was completed with a frit array where the $\mathrm{Na}_{2} \mathrm{O}$ concentration was either 0 or $1 \mathrm{wt} \%$. The results of the Nominal Stage assessment identified 71 frits from this array that provided projected operating windows of at least 30-40\% WL. Six of these frits, described in Table 3-51, were down-selected for a Variation Stage assessment using the same EVs.

Table 3-51. Frit Compositions with $\mathrm{No} \mathrm{Na}_{2} \mathrm{O}$ Selected for a Variation Stage Assessment.

\begin{tabular}{||c|c|c|c||}
\hline $\mathbf{B}_{\mathbf{2}} \mathbf{O}_{\mathbf{3}}$ & $\mathbf{L i}_{\mathbf{2}} \mathbf{O}$ & $\mathbf{N a}_{\mathbf{2}} \mathbf{O}$ & $\mathbf{S i O}_{\mathbf{2}}$ \\
\hline 12 & 10 & 0 & 78 \\
\hline 12 & 12 & 0 & 76 \\
\hline 14 & 10 & 0 & 76 \\
\hline 14 & 11 & 0 & 75 \\
\hline 10 & 12 & 0 & 78 \\
\hline 8 & 12 & 0 & 80 \\
\hline
\end{tabular}

The outcome of the Variation Stage assessment is described in Table 3-52. The results for the centroid - or average - of the sludge composition region are given in the first row of the table. Note that relatively large projected operating windows are available, with waste loadings up to $47 \%$. The results for the assessment with variation included are given in the bottom row of the table. The projected operating windows are significantly reduced for each frit when variation is considered, indicating that the $\mathrm{Na}_{2} \mathrm{O}$ concentrations in these systems are in a difficult processing region and making these frits poor candidates for SB6 vitrification. 
Table 3-52. Variation Stage Results for Frits Containing $\mathrm{No} \mathrm{Na}_{2} \mathrm{O}$.

\begin{tabular}{|c|c|c|c|c|c|c|}
\hline & B-12;Li-10;Si-78 & B-12;Li-12;Si-76 & B-14;Li-10;Si-76 & B-14;Li-11;Si-75 & B-10;Li-12;Si-78 & B-8;Li-12;Si-80 \\
\hline Centroid & $\begin{array}{c}- \\
25-45 \\
\mathrm{~T}_{\mathrm{L}} \\
47\end{array}$ & $\begin{array}{c}- \\
25-44 \\
\text { lowv } \\
47\end{array}$ & $\begin{array}{c}- \\
25-45 \\
\mathrm{~T}_{\mathrm{L}} \\
47\end{array}$ & $\begin{array}{c}- \\
25-45 \\
\text { lowv, Neph } \\
46\end{array}$ & $\begin{array}{c}- \\
25-46 \\
\text { Neph } \\
47\end{array}$ & $\begin{array}{c}- \\
25-47 \\
\text { Neph } \\
48\end{array}$ \\
\hline Variation (EVs) & $\begin{array}{c}\text { highv } \\
32-36 \\
\mathrm{~T}_{\mathrm{L}} \\
45\end{array}$ & $\begin{array}{c}- \\
25-38 \\
\text { lowv, } T_{L} \\
44\end{array}$ & $\begin{array}{c}\text { highv } \\
26-36 \\
\mathrm{~T}_{\mathrm{L}} \\
44\end{array}$ & $\begin{array}{c}- \\
25-37 \\
\mathrm{~T}_{\mathrm{L}} \\
44\end{array}$ & $\begin{array}{c}- \\
25-39 \\
\mathrm{~T}_{\mathrm{L}} \\
45\end{array}$ & $\begin{array}{c}\text { highv } \\
26-39 \\
\mathrm{~T}_{\mathrm{L}} \\
45\end{array}$ \\
\hline
\end{tabular}


SRNL-STI-2010-00137

Revision 0

\subsection{Summary}

A brief summary of each set of paper study assessments is given below, divided into the same time periods used in the previous section.

\section{November 2009 SB6 Composition Projections}

- Nominal Stage projected operating windows were limited to $40-44 \%$ WL for candidate frits for both the SRR and SRNL SB6 projections.

- Low $\mathrm{Na}_{2} \mathrm{O}$ concentration frits were limited by $\mathrm{T}_{\mathrm{L}}$ or low viscosity predictions.

- Higher $\mathrm{Na}_{2} \mathrm{O}$ concentration frits were limited by nepheline crystallization predictions.

- When variation was accounted for, the projected operating windows for the candidate frits were significantly reduced.

- They remained limited by $\mathrm{T}_{\mathrm{L}}$ predictions, with maximum waste loadings of $35 \%$.

- Frit 418 was the best performer, with all EVs acceptable over waste loadings of $25-37 \%$, limited by $\mathrm{T}_{\mathrm{L}}$ predictions.

○ However, this would leave little room for error in targeting 36\% WL for SB6.

Early December 2009 SB6 Composition Projections

- SRNL revised its SB6 composition projections to account for a larger decant at the end of the washing process in Tank 51.

- A large number of frits were available that provided projected operating windows of at least $28-40 \%$ WL at the Nominal Stage.

- Frit 418 was limited by $\mathrm{T}_{\mathrm{L}}$ predictions at about $40 \% \mathrm{WL}$.

- Alternative frits could provide $42 \% \mathrm{WL}$ at Nominal Stage, and were also limited by $\mathrm{T}_{\mathrm{L}}$ predictions.

- With variation included, maximum waste loadings were reduced to $36-38 \%$.

- Frit 418 was $\mathrm{T}_{\mathrm{L}}$ limited at $37 \% \mathrm{WL}$.

$\circ$ There was no practical change in predicted performance for Frit 418 with these SB6 projections, and a slight improvement for alternative frits.

Mid-December 2009 SB6 Composition Projections

- These projections focused on uncertainty in the blending of SB6, or the timing of the transfer from Tank 51 to Tank 40.

- SRNL studied three blend points and two washing scenarios.

- The impact of changes in blend point was minimal in terms of frit optimization.

- The predicted performance of Frit 418 at the Nominal Stage showed no practical difference for the various blend scenarios.

Late December 2009 SB6 Composition Projections

- SRR provided guidance that the transfer from Tank 51 to Tank 40 may have to occur earlier, necessitating a decant in Tank 40 during SB6 processing.

$\circ$ In other words, the SB6 composition would change after a few weeks of processing.

- The number of frits available that provide reasonable projected operating windows was significantly reduced after the decant in Tank 40.

- 1148 frits compositions were available prior to the decant, and 175 frit compositions were available after.

- The performance of Frit 418 at the Nominal Stage was reduced after the Tank 40 decant, with the maximum waste loading now $38 \%$. 
- Alternative frits could get to $41 \% \mathrm{WL}$ after the Tank 40 decant, which would be limited by $T_{L}$ predictions, although these compositions would not be viable prior to decant.

- A Variation Stage assessment was not performed due to uncertainty in the Tank 40 decant plans.

\section{Early January 2010 SB6 Composition Projections}

- Further revisions in the SB6 washing strategy were received from SRR.

$\circ$ These projections again included scenarios before and after a decant in Tank 40.

- There was a slight improvement in the predicted performance of Frit 418 with these projections.

- Maximum waste loadings were $41 \%$ before the Tank 40 decant (limited by nepheline crystallization predictions) and $40 \%$ after the decant (limited by $\mathrm{T}_{\mathrm{L}}$ predictions).

- A search for other candidate frits that could provide viable projected operating windows yielded 275 potential frits with a maximum waste loading of $46 \%$ before the decant, and 84 potential frits with a maximum waste loading of $43 \%$ after the decant.

- However, the number of candidate frits that could provide reasonable projected operating windows both before and after the Tank 40 decant was very limited, with Frit 418 being one of the best performers.

$\circ$ With variation included, the projected operating window where all the EVs were predicted to be acceptable for Frit 418 had a maximum waste loading of $39 \%$ before the decant and $35 \%$ after the decant.

- This continued to leave little room for error in targeting 36\% WL for SB6.

- A series of additional frit modifications was explored to look for other routes to improve projected operating windows.

- Higher $\mathrm{Na}_{2} \mathrm{O}$ concentration frits provided no practical improvement, using the predicted performance of Frit 418 as a benchmark.

- Frits similar in composition to Frit 418 but with slightly higher alkali concentrations again provided no practical improvement.

$\circ$ Lower $\mathrm{Na}_{2} \mathrm{O}$ concentration frits were found to provide a slight improvement in projected operating windows, but only for the pre-decant composition projection.

$\circ$ Higher $\mathrm{B}_{2} \mathrm{O}_{3}$ concentration frits provided no practical improvement.

- After these iterations, there was no clear path to improving on the predicted performance of Frit 418 for these SB6 composition projections.

- The change in sludge $\mathrm{Na}_{2} \mathrm{O}$ concentration after the Tank 40 decant makes it difficult to identify a single frit composition that will perform well both before and after the decant.

- If the sodium concentration could be adjusted after the decant (e.g., an addition of $\mathrm{NaOH}$ to Tank 40) it may provide an opportunity for improved frit performance.

- A significant improvement in the Variation Stage performance of Frit 418 after the Tank 40 decant was noted when the sludge $\mathrm{Na}_{2} \mathrm{O}$ concentration was increased.

Late January 2010 SB6 Composition Projections

- The SB6 composition projections before and after a decant in Tank 40 were further revised.

- Many candidate frits were available prior to the decant, but only 44 frits were available that could provide projected operating windows of at least $30-40 \% \mathrm{WL}$ after the decant, with a maximum waste loading of $41 \%$. 
- A larger array of 353,430 frit compositions was explored, including additional components such as $\mathrm{CaO}$ and $\mathrm{Fe}_{2} \mathrm{O}_{3}$.

- 234 frits were available from this larger array that could provide projected operating windows of at least $30-40 \%$ WL after the decant, although there was no improvement in the maximum projected waste loading of $41 \%$.

February 2010 SB6 Composition Projections

- SRNL revised the SB6 composition projections using the measured qualification sample composition, and included several potential blend points.

○ Note that these projections did not include the impact of a decant of Tank 40 during SB6 processing.

- At the Nominal Stage, Frit 418 was limited by nepheline crystallization predictions at $41 \% \mathrm{WL}$ for all the projections.

- Blend point again had no practical impact on the projected performance of Frit 418.

- Frit 418 was limited by nepheline crystallization predictions at 38\% WL when variation was considered.

○ Other frits were available that could get to $40 \% \mathrm{WL}$ with variation considered and move nepheline crystallization predictions up to $43-44 \%$ WL. However, these other frits have low $\mathrm{Na}_{2} \mathrm{O}$ concentrations that could potentially hinder melt rate.

- Additional candidate frits are available with higher $\mathrm{Na}_{2} \mathrm{O}$ concentrations, with predicted performance similar to that of Frit 418.

\subsection{Recommendations}

Based on the most recent, February 2010 SB6 composition projections developed at SRNL, Frit 418 appears to be viable for SB6 processing at a target waste loading of $36 \%$. This assessment is made from a paper study approach only, and assumes that no decant of Tank 40 will occur during SB6 processing. A decant of Tank 40 would reduce the $\mathrm{Na}_{2} \mathrm{O}$ concentration in Tank 40 to a point where it would be very difficult to target a waste loading of 36\% for SB6 with Frit 418 , or any other frit studied. These recommendations were also included in recent memorandum discussing the use of Frit 418 for SB6 vitrification. ${ }^{1}$

The performance of Frit 418 with SB6 is limited by predictions of nepheline crystallization, which is a waste form affecting constraint. Alternatives to Frit 418 are available that can provide equivalent projected operating windows and are limited by process related constraints (i.e., liquidus temperature predictions) rather than durability related constraints. Potential differences in melt rate among these alternative frits will be discussed in a forthcoming report.

\subsection{References}

1. Fox, K. M. and T. B. Edwards, "Glass Frit Composition for Sludge Batch 6 Vitrification at the Defense Waste Processing Facility," U.S. Department of Energy Report SRNL-L3100-201000043, Savannah River National Laboratory, Aiken, SC (2010).

2. Fox, K. M. and T. B. Edwards, "Recommended Glass Frit for Vitrification of the SB6 Qualification Sample," U.S. Department of Energy Report SRNL-L3100-2010-00043, Savannah River National Laboratory, Aiken, SC (2010). 
3. Bricker, J. M., "Technical Task Request: Sludge Batch 6 Frit Optimization," U.S. Department of Energy Report HLW-DWPF-TTR-2009-0002, Revision 0, Washington Savannah River Company, Aiken, SC (2008).

4. Fox, K. M., "Task Technical and Quality Assurance Plan: Sludge Batch 6 Frit Optimization,” U.S. Department of Energy Report SRNL-RP-2008-01342, Revision 1, Savannah River National Laboratory, Aiken, SC (2009).

5. JMP ${ }^{\mathrm{TM}}$, Ver. 7.0.2, [Computer Software] SAS Institute Inc., Cary, NC (2007).

6. Bannochie, C. J., J. M. Pareizs and D. R. Click, "Tank 51 Qualification SRAT Receipt Characterization," U.S. Department of Energy Report SRNL-L3100-2010-00027, Savannah River National Laboratory, Aiken, SC (2010).

7. Lambert, D. P., D. H. Miller, D. K. Peeler, M. E. Smith and M. E. Stone, "Impact of Alkali Source on Vitrification of SRS High Level Waste," U.S. Department of Energy Report WSRCMS-2005-00319, Washington Savannah River Company, Aiken, SC (2005). 


\section{Distribution:}

C. J. Bannochie, 773-42A

A. B. Barnes, 999-W

A. L. Billings, 999-W

J. M. Bricker, 704-27S

D. A. Crowley, 773-43A

T. B. Edwards, 999-W

T. L. Fellinger, 704-26S

S. D. Fink, 773-A

K. M. Fox, 999-W

B. J. Giddings, 786-5A

J. M. Gillam, 766-H

B. A. Hamm, 766-H

C. C. Herman, 999-W

E. W. Holtzscheiter, 704-15S

J. F. Iaukea, 704-30S

F. C. Johnson, 999-W

M. T. Keefer, 766-H

S. L. Marra, 773-A

R. T. McNew, 704-27S

A. M. Murray, 773-A

J. E. Occhipinti, 704-S

D. K. Peeler, 999-W

F. M. Pennebaker, 773-42A

H. M. Pittman, 704-27S

J. W. Ray, 704-S

H. B. Shah, 766-H

D. C. Sherburne, 704-S

M. E. Smith, 704-30S

M. E. Stone, 999-W

W. R. Wilmarth, 773-A

J. R. Zamecnik, 999-W 